CRM-3182 (2005)

\title{
Biorthogonal Laurent polynomials, Töplitz determinants, minimal Toda orbits and isomonodromic tau functions
}

\author{
M. Bertola ${ }^{12}$, M. Gekhtman ${ }^{34}$
}

\begin{abstract}
We consider the class of biorthogonal polynomials that are used to solve the inverse spectral problem associated to elementary co-adjoint orbits of the Borel group of upper triangular matrices; these orbits are the phase space of generalized integrable lattices of Toda type. Such polynomials naturally interpolate between the theory of orthogonal polynomials on the line and orthogonal polynomials on the unit circle and tie together the theory of Toda, relativistic Toda, Ablowitz-Ladik and Volterra lattices. We establish corresponding Christoffel-Darboux formulæ. For all these classes of polynomials a $2 \times 2$ system of Differential-Difference-Deformation equations is analyzed in the most general setting of pseudo measures with arbitrary rational logarithmic derivative. They provide particular classes of isomonodromic deformations of rational connections on the Riemann sphere. The corresponding isomonodromic tau function is explicitly related to the shifted Töplitz determinants of the moments of the pseudo-measure. In particular the results imply that any (shifted) Töplitz (Hänkel) determinant of a symbol (measure) with arbitrary rational logarithmic derivative is an isomonodromic tau function.
\end{abstract}

\section{Contents}

\begin{tabular}{lll}
\hline & Introduction & 2
\end{tabular}

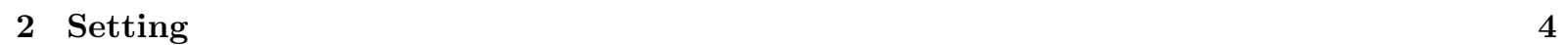

2.1 Second-kind polvnomials . . . . . . . . . . . . . . . . . . . . . 6

3 Christoffel-Darboux formulæ 6

3.1 Principal CDI . . . . . . . . . . . . . . . . . . . . . . . . 8

4 CDIs for biorthogonal Laurent polynomials

5 Infinitesimal deformations of the moment functional 11

5.1 Deformations for the second-kind (Laurent) polvnomials . . . . . . . . . . . . . . . . . . 13

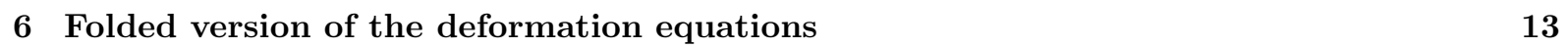

7 Moment functionals of integral type and ODE 16

7.1 Differential equations . . . . . . . . . . . . . . . . . . . . . . . . 18

\footnotetext{
${ }^{1}$ Work supported in part by the Natural Sciences and Engineering Research Council of Canada (NSERC), Grant. No. 261229-03 and by the Fonds FCAR du Québec.

${ }^{2}$ e-mail: bertola@mathstat.concordia.ca

${ }^{3}$ Work supported in part by the National Science Foundation, Grant No. DMS-0400484.

${ }^{4}$ e-mail: gekhtman.1@nd.edu
} 
8 Spectral curve and Isomonodromic tau function $\quad 20$

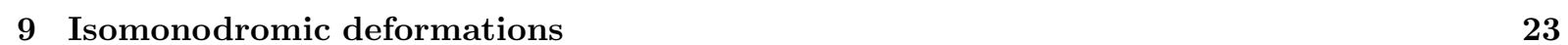

9.1 Spectral residue-formulæ $\ldots \ldots \ldots \ldots \ldots \ldots \ldots \ldots$

10 Riemann-Hilbert problem. Tau function $\quad 26$

10.1 Isomonodromic Tau Function . . . . . . . . . . . . . . . . . . . . . . 27

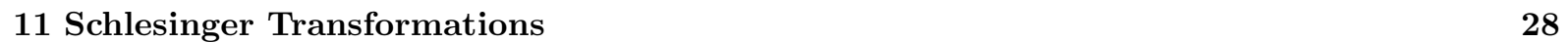

$\begin{array}{ll}12 \text { Conclusion } & 29\end{array}$

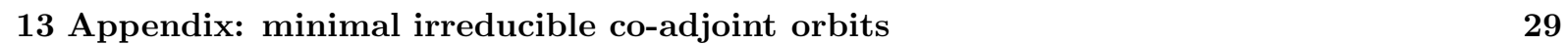

\section{Introduction}

The connection between orthogonal polynomials on the line and Toda lattices is rather well known [3, as well as the relations to the KP hierarchy [1. Dynamical variables of the Toda lattice are arranged into a tri-diagonal Lax matrix, that can be viewed as a recurrence matrix for a system of orthogonal polynomials. In the (semi)finite case, the evolution of the corresponding measure provides a linearization of the Toda flows. More generally, one can set-up (in)finite-dimensional Hamiltonian systems on $\mathbb{R}^{2 n}(n \leq \infty)$ with Hamiltonians

$$
\begin{array}{r}
H_{I}(\underline{q}, \underline{p})=\frac{1}{2} \sum_{i=1}^{n} p_{i}{ }^{2}+\sum_{i \notin I} p_{i} \mathrm{e}^{q_{i+1}-q_{i}}+\sum_{j=1}^{|I|} \mathrm{e}^{q_{i_{j+1}}-q_{i_{j}}} \\
I:=\left\{i_{1}<i_{2}<\ldots<i_{k}\right\} .
\end{array}
$$

As it is noted in [10] such family of Hamiltonians (labeled by the multi-index $I$ ) contains integrable lattice hierarchies of Toda, relativistic Toda, Volterra and Ablowitz-Ladik type. These integrable Hamiltonian systems have a Lax representation with Lax operator given as a $n \times n$ lower Hessenberg matrix which we denote by $Q$ (in [9, 10] it was denoted by $X$ ), belonging to a certain "elementary" $(2 n-2)$-dimensional co-adjoint orbit of the solvable group of upper triangular matrices. These systems are linearized by the Moser map

$$
\begin{array}{r}
Q \mapsto \mathcal{W}(z ; Q):=(z \mathbf{1}-Q)_{11}^{-1}=\sum_{j=0}^{\infty} \frac{\hat{\mu}_{j}(Q)}{z^{j+1}} \\
\hat{\mu}_{j}(Q)=Q^{j}{ }_{11}
\end{array}
$$

In the case of infinite lattices these expressions take on a formal meaning in terms of power series but the analysis is unchanged.

The moments $\hat{\mu}_{j}$ of $Q$ define a normalized moment functional $\mathcal{L}$ and the reconstruction of $Q$ from its moments (the "inverse moment problem") can be accomplished by constructing a suitable sequence of biorthogonal (Laurent) polynomials $\left\{r_{i}, p_{i}\right\}_{i \in \mathbb{N}}$

$$
\mathcal{L}\left(r_{i} p_{j}\right)=\delta_{i j},
$$

where $p_{i}$ 's are polynomials in $x$ of degree $i$ while $r_{i}$ 's are, in general, polynomials in $x$ and $x^{-1}$. The (infinite) Lax operator $Q$ corresponding to the chosen orbit is then reconstructed by [10]

$$
Q_{i j}=\mathcal{L}\left(r_{i} x p_{j}\right) .
$$


Explicit formulæ for these biorthogonal polynomials in terms of shifted Töplitz determinants can be found in [9, 10] and will be recalled here in due time. Vice versa, one could assign an arbitrary (generic) moment functional $\mathcal{L}: \mathbb{C}\left[z, z^{-1}\right] \rightarrow \mathbb{C}$, a multi-index $I$ and then reconstruct the Lax operator $Q_{I}$ (i.e. view the Lax operator as a function of $\mathcal{L}$ rather than the other way around)

$$
\mathcal{L} \mapsto Q_{I}(\mathcal{L})
$$

From this point of view, the linearization of the (infinite) Hamiltonian hierarchy is accomplished simply by

$$
\mathcal{L}_{\mathbf{t}}(\bullet)=\mathcal{L}\left(\mathrm{e}^{\sum_{i} 1 / i t_{i} z^{i}} \bullet\right)
$$

where the series may have to be understood formally. This procedure displays the common nature of all the above-mentioned integrable lattices, inasmuch as the linearizing space is always the same (the space of moment functionals) and what changes from one lattice to another is only the orbit, namely the map $Q_{I}$.

Finite dimensional systems (of dimension $2 n-2$ ) on an elementary orbit $\mathfrak{Q}_{I}$ correspond to those moment-functionals for which certain shifted Töplitz determinants of size $\leq n$ do not vanish whereas all larger ones do. In such cases, the tau function of the hierarchy defined by the (closed) differential

$$
\mathrm{d} \ln \tau=\sum_{J=1}^{n} \frac{1}{J} \operatorname{Tr}_{n}\left(Q_{I}\right) \mathrm{d} t_{J}
$$

and coincides with the largest non-vanishing (shifted) Töplitz determinant.

One of the main purposes of this paper is to connect this determinant to a different notion of "tau" function, namely the one introduced by Jimbo, Miwa and Ueno in 13, 14. It was shown in 6, 4] that the Hänkel determinants of an arbitrary (generic) "semiclassical" moment functional on the space of polynomials can be identified with the isomonodromic tau function introduced by our Japanese colleagues. Similarly, it was shown in [16 that Töplitz determinants of a particular class of symbols on the unit circle are also identifiable with the same kind of isomonodromic tau functions.

These two apparently distinct situations are in fact the two ends of a "continuous" spectrum of situations: in fact the case of Hänkel determinants is dealt with in the setting of (generalized) ordinary orthogonal polynomials, whereas that of Töplitz determinants uses orthogonal polynomials on the unit circle; in this latter situation one considers polynomials $p_{i}(z)$ orthogonal in the usual $L^{2}\left(S^{1}, \mathrm{~d} \mu\right)$ sense

$$
\int_{S^{1}} p_{j}(z) \overline{p_{k}(z)} \mathrm{d} \mu(z)=\delta_{j k} .
$$

Here one defines $r_{j}(z)=\overline{p_{j}}\left(z^{-1}\right)$ and the orthogonality is recast into

$$
\mathcal{L}\left(r_{j} p_{k}\right)=\delta_{j k},
$$

where - in this special case -

$$
\mathcal{L}: \mathbb{C}\left[z, z^{-1}\right] \rightarrow \mathbb{C} ; \quad \mathcal{L}\left(z^{j}\right)=\int_{S^{1}} z^{j} \mathrm{~d} \mu(z) .
$$

We see that we can regard the case of orthogonal polynomials on the circle as a special case of biorthogonal Laurent polynomials with respect to a moment functional satisfying the reality condition $\mu_{k}=\overline{\mu_{-k}}$.

According to the previous description of integrable lattices, the two situations correspond to two different elementary orbits and hence we should be able to treat them on a common ground, together with all the other lattices associated with the orbits $\mathfrak{Q}_{I}$. Indeed, we will show that this is the case and that for the class of moment functionals of the semiclassical type introduced in [4] all the shifted Töplitz 
determinants which arise as tau functions of the corresponding integrable lattices are also isomonodromic tau functions for a rational $2 \times 2$ connection on $\mathbb{C}^{1}$ which will be explicitly constructed in the paper.

The approach to this problem follows the strategy used in [4] rather the one in [16]; in the course of our analysis we will obtain generalized Christoffel-Darboux identities which naturally interpolate between the ordinary CD identity for orthogonal polynomials on the line and the one for orthogonal polynomials on the unit circle.

Moreover we will show that the Töplitz and Hänkel determinants of the same size for one such moment functional are connected by a sequence of elementary Schlesinger transformations, at each step of which we obtain tau functions associated to interpolating orbits; in figurative terms, we show that the papers [16] (see Example 10.1) and 4] are connected by a Schlesinger transformation (when specializing the semiclassical measure to the one relevant for [16]) and that "neighboring" elementary co-adjoint orbits are also connected by an elementary Schlesinger transformation.

Acknowledgements. The authors thank John Harnad for stimulating discussions. M. G. is grateful to Laboratoire de Physique Mathematique, Centre de Recherches Mathematique and the Concordia University for their hospitality during his visit to Montreal, where the work on this project has started. During the later stages of preparation of the manuscript he also enjoyed hospitality of Institut des Hautes Études Scientifiques.

\section{Setting}

We start in the most general and abstract setting, without any reference to a (pseudo) measure. We consider an arbitrary moment functional

$$
\mathcal{L}: \mathbb{C}\left[z, z^{-1}\right] \rightarrow \mathbb{C}
$$

on the space polynomials in $z$ and $z^{-1}$ and denote its moments with $\mu_{j}=\mathcal{L}\left(z^{j}\right), j \in \mathbb{Z}$. We introduce the following shifted Töplitz determinants and polynomials

$$
\begin{array}{r}
\Delta_{n}^{\ell}=\operatorname{det}\left(\begin{array}{cccc}
\mu_{\ell} & \mu_{\ell+1} & \cdots & \mu_{\ell+n-1} \\
\mu_{\ell-1} & \mu_{\ell} & \cdots & \mu_{\ell+n-2} \\
& \ddots & \ddots & \\
\mu_{\ell-n+1} & \mu_{\ell-n+2} & \cdots & \mu_{\ell}
\end{array}\right) \\
\wp_{n}^{\ell}(x):=\operatorname{det}\left(\begin{array}{cccc}
\mu_{\ell} & \mu_{\ell+1} & \cdots & \Delta_{-n}^{\ell} \equiv 0 \\
\mu_{\ell-1} & \mu_{\ell} & \cdots & \mu_{\ell+n-1} \\
& \ddots & \ddots & \\
\mu_{\ell-n+1} & \mu_{\ell-n+2} & \cdots & \mu_{\ell+1} \\
1 & x & \cdots & x^{n}
\end{array}\right)
\end{array}
$$

Using some classical identities for determinants we can derive recurrence relations for the shifts $n \rightarrow$ $n+1$ and $\ell \rightarrow \ell+1$ for the above polynomials. We first need the following

Proposition 2.1 For any $(n+1) \times(n+1)$ matrix $A$ the following determinant identity holds true (Jacobi identity)

$$
A_{1 . . n}^{1 . . n} A_{2 . . n+1}^{2 . . n+1}-A_{1 . . n}^{2 . . n+1} A_{2 . . n+1}^{1 . . n}=A_{1 . . n+1}^{1 . . n+1} A_{2 . . n}^{2 . . n},
$$

where the sub/super-script ranges denote the rows/columns of the submatrix we are computing the determinant of. As a corollary, for any $(n+1) \times(n+2)$ matrix $B$ we have

$$
B_{2 . . n+2}^{1 . . n+1} B_{1 . . n}^{1 . . n}+B_{1 . . n+1}^{1 . n+1} B_{2 . . n, n+2}^{1 . . n}=B_{1 . . n, n+2}^{1 . . n+1} B_{2 . . n+1}^{1 . . n}
$$

which can be obtained from (2-4) by adjoining an appropriate row. 
Using (2-4) on the determinant defining $\wp_{n}^{\ell}$ we find

$$
x \Delta_{n}^{\ell} \wp_{n-1}^{\ell}-\Delta_{n}^{\ell+1} \wp_{n-1}^{\ell-1}=\Delta_{n-1}^{\ell} \wp_{n}^{\ell} .
$$

Applying (2-5) to the determinant defining $\wp_{n}^{\ell}$ adjoined of the next row of moments on the top we find

$$
\begin{aligned}
& \wp_{n}^{\ell-1} \Delta_{n}^{\ell}+\Delta_{n+1}^{\ell} \wp_{n-1}^{\ell-1}=\wp_{n}^{\ell} \Delta_{n}^{\ell-1} \\
& \wp_{n}^{\ell-1} \Delta_{n}^{\ell+1}+x \Delta_{n+1}^{\ell} \wp_{n-1}^{\ell}=\wp_{n}^{\ell} \Delta_{n}^{\ell} \\
& x \Delta_{n}^{\ell} \wp_{n-1}^{\ell}-\Delta_{n}^{\ell+1} \wp_{n-1}^{\ell-1}=\wp_{n}^{\ell} \Delta_{n-1}^{\ell}
\end{aligned}
$$

We now use these identities to express $\wp_{n}^{\ell}:=\left[\wp_{n}^{\ell}, \wp_{n-1}^{\ell-1}\right]$ in terms of $\wp_{n-1}^{\ell}=\left[\wp_{n-1}^{\ell}, \wp_{n-2}^{\ell-1}\right]$

$$
\begin{aligned}
& {\left[\begin{array}{c}
\wp_{n}^{\ell} \\
\wp_{n-1}^{\ell-1}
\end{array}\right]=\left[\begin{array}{cc}
\frac{x \Delta_{n}^{\ell}}{\Delta_{n-1}^{\ell}}-\frac{\Delta_{n}^{\ell+1} \Delta_{n-1}^{\ell-1}}{\left(\Delta_{n-1}^{\ell}\right)^{2}} & \frac{\Delta_{n}^{\ell+1} \Delta_{n}^{\ell}}{\left(\Delta_{n-1}^{\ell}\right)^{2}} \\
\frac{\Delta_{n-1}^{\ell-1}}{\Delta_{n-1}^{\ell}} & -\frac{\Delta_{n}^{\ell}}{\Delta_{n-1}^{\ell}}
\end{array}\right]\left[\begin{array}{c}
\wp_{n-1}^{\ell} \\
\wp_{n-2}^{\ell-1}
\end{array}\right]} \\
& \wp_{n}^{\ell}=\mathcal{C}_{n}^{\ell} \wp_{n-1}^{\ell} \\
& \operatorname{det} \mathcal{C}_{n}^{\ell}=-x \frac{\left(\Delta_{n}^{\ell}\right)^{2}}{\left(\Delta_{n-1}^{\ell}\right)^{2}} \\
& \mathbf{j} \mathcal{C}_{n}^{\ell}(x)^{-1} \mathcal{C}_{n}^{\ell}(y)-\mathbf{j}=\left(1-\frac{y}{x}\right)\left[\begin{array}{cc}
\frac{\Delta_{n-1}^{\ell-1}}{\Delta_{n}^{\ell}} & 0 \\
-1 & 0
\end{array}\right]
\end{aligned}
$$

where

$$
\mathbf{j}:=\left[\begin{array}{cc}
0 & 1 \\
-1 & 0
\end{array}\right]
$$

We have named this the "circle case" because this sort of recursion is relevant for orthogonal polynomials on the unit circle. We next derive a recursion in $\ell$

$$
\begin{aligned}
& {\left[\begin{array}{c}
\wp_{n}^{\ell} \\
\wp_{n-1}^{\ell-1}
\end{array}\right]=\left[\begin{array}{cc}
\frac{\Delta_{n}^{\ell}}{\Delta_{n}^{\ell-1}}+\frac{\Delta_{n+1}^{\ell} \Delta_{n-1}^{\ell-1}}{x\left(\Delta_{n}^{\ell-1}\right)^{2}} & \frac{\Delta_{n+1}^{\ell} \Delta_{n}^{\ell}}{x\left(\Delta_{n}^{\ell-1}\right)^{2}} \\
\frac{\Delta_{n-1}^{\ell-1}}{x \Delta_{n}^{\ell-1}} & \frac{\Delta_{n}^{\ell}}{x \Delta_{n}^{\ell-1}}
\end{array}\right]} \\
& \wp_{n}^{\ell}=\mathcal{T}_{n}^{\ell} \wp_{n}^{\ell-1} \\
& \operatorname{det} \mathcal{T}_{n}^{\ell}=\frac{1}{x} \frac{\left(\Delta_{n}^{\ell}\right)^{2}}{\left(\Delta_{n}^{\ell-1}\right)^{2}} \\
& \mathbf{j} \mathcal{T}_{n}^{\ell}(x)^{-1} \mathcal{T}_{n}^{\ell}(y)-\mathbf{j}=\left(1-\frac{x}{y}\right)\left[\begin{array}{c}
\wp_{n}^{\ell-1} \\
\wp_{n-1}^{\ell-2}
\end{array}\right]
\end{aligned}
$$

The name "circle-to-line" refers to the fact that this recursion relation interpolates between the previous "circle" case and the next one, which will be named the "line" case. Indeed, composing these two we can 
express $\wp_{n}^{\ell}=\left[\wp_{n}^{\ell}, \wp_{n-1}^{\ell-1}\right]$ in terms of $\wp_{n-1}^{\ell-1}=\left[\wp_{n-1}^{\ell-1}, \wp_{n-2}^{\ell-2}\right]$

$$
\begin{aligned}
& {\left[\begin{array}{c}
\wp_{n}^{\ell} \\
\wp_{n-1}^{\ell-1}
\end{array}\right]=\left[\begin{array}{cc}
\frac{\Delta_{n}^{\ell}}{\Delta_{n-1}^{\ell-1}}\left(x+\frac{\Delta_{n-2}^{\ell-1} \Delta_{n}^{\ell}-\Delta_{n}^{\ell+1} \Delta_{n-1}^{\ell-1}}{\Delta_{n-1}^{\ell} \Delta_{n-1}^{\ell-1}}\right) & \frac{\left(\Delta_{n}^{\ell}\right)^{2}}{\left(\Delta_{n-1}^{\ell-1}\right)^{2}} \\
1 & 0
\end{array}\right]\left[\begin{array}{c}
\wp_{n-1}^{\ell-1} \\
\wp_{n-2}^{\ell-2}
\end{array}\right]} \\
& \wp_{n}^{\ell}=\mathcal{L}_{n}^{\ell} \wp_{n-1}^{\ell-1} \\
& \operatorname{det} \mathcal{L}_{n}^{\ell}=-\frac{\left(\Delta_{n}^{\ell}\right)^{2}}{\left(\Delta_{n-1}^{\ell-1}\right)^{2}} \\
& \mathbf{j} \mathcal{L}_{n}^{\ell}(x)^{-1} \mathcal{L}_{n}^{\ell}(y)-\mathbf{j}=(x-y)\left[\begin{array}{cc}
\frac{\Delta_{n-1}^{\ell-1}}{\Delta_{n}^{\ell}} & 0 \\
0 & 0
\end{array}\right]
\end{aligned}
$$

This recursion is called "line" case because it is the relevant recursion relation for ordinary orthogonal polynomials on the line.

\subsection{Second-kind polynomials}

Let us define the following second-kind polynomials

$$
\mathcal{R}_{n}^{\ell}(x)=\mathcal{L}_{z}\left(\frac{\wp_{n}^{\ell}(x)-\wp_{n}^{\ell}(z)}{x-z}\right)
$$

The three types of recursion (2-10, 2-15 2-19) involve at most a multiplication or division by $x$ and have otherwise constant coefficients (in $x$ ): moreover we find

$$
\begin{aligned}
x \mathcal{R}_{n}^{\ell}(x) & =\mathcal{L}_{z}\left(\frac{x \wp_{n}^{\ell}(x)-z \wp_{n}^{\ell}(z)}{x-z}\right)-\mathcal{L}_{z}\left(\wp_{n}^{\ell}(z)\right) \\
x^{-1} \mathcal{R}_{n}^{\ell}(x) & =\mathcal{L}_{z}\left(\frac{x^{-1} \wp_{n}^{\ell}(x)-z^{-1} \wp_{n}^{\ell}(z)}{x-z}\right)-\frac{1}{x} \mathcal{L}_{z}\left(z^{-1} \wp_{n}^{\ell}(z)\right)
\end{aligned}
$$

The last terms in these identities vanish because of the determinant structure of $\wp_{n}^{\ell}$, provided that $n \geq 1$ and $0 \leq \ell \leq n-1$ for the first case and $-1 \leq \ell \leq n-2$ for the second case. From this observation we find that these auxiliary sequences of polynomials satisfy the same recurrence relations in the following ranges

$$
\left[\begin{array}{c}
\mathcal{R}_{n}^{\ell} \\
\mathcal{R}_{n-1}^{\ell-1}
\end{array}\right]=\left\{\begin{array}{cc}
\mathcal{L}_{n}^{\ell}\left[\begin{array}{l}
\mathcal{R}_{n-1}^{\ell-1} \\
\mathcal{R}_{n-2}^{\ell-2}
\end{array}\right] & 1 \leq \ell \leq n-1 \\
\mathcal{C}_{n}^{\ell}\left[\begin{array}{c}
\mathcal{R}_{n-1}^{\ell} \\
\mathcal{R}_{n-2}^{\ell-1}
\end{array}\right] & 0 \leq \ell \leq n-2 \\
\mathcal{T}_{n}^{\ell}\left[\begin{array}{l}
\mathcal{R}_{n-1}^{\ell-1} \\
\mathcal{R}_{n-2}^{\ell-2}
\end{array}\right] & 0 \leq \ell \leq n-1
\end{array}\right.
$$

\section{Christoffel-Darboux formulæ}

Consider $(n, l) \in \mathbb{N} \times \mathbb{N}$ and choose an arbitrary path starting at the origin of the following type

$$
\left\{\left(n_{k}, \ell_{k}\right), k=0,1, \ldots,\left(n_{0}, \ell_{0}\right)=(0,0),\left(n_{1}, \ell_{1}\right)=(1,0)\right\}
$$


and such that the possible subsequent moves are right, up or up-right. For the move $\left(n_{k-1}, \ell_{k-1}\right) \mapsto\left(n_{k} \ell_{k}\right)$ we introduce the transfer matrices following an idea of [12] used for orthogonal polynomials on the circle

$$
T_{k}(x):= \begin{cases}\mathcal{C}_{n_{k}}^{\ell_{k}} & \text { if the move is right (circle move) } \\ \mathcal{T}_{n_{k}}^{\ell_{k}} & \text { if the move is up (circle-to-line move) } \\ \mathcal{L}_{n_{k}}^{\ell_{k}} & \text { if the move is up-right (line move) }\end{cases}
$$

Using these transfer matrices we define the two dual auxiliary sequences of matrices as follows

$$
\begin{aligned}
& \Xi_{k}(x)=T_{k}(x) \Xi_{k-1}(x) \\
& \Xi_{k}^{\star}(x)=\frac{1}{\operatorname{det} T_{k}(x)} \Xi_{k-1}^{\star}(x) T_{k}^{t}(x) \\
& \Xi_{0}^{\star}=\Xi_{0}^{t} .
\end{aligned}
$$

This definition in particular implies that

$$
\Xi_{k}^{\star}=\frac{1}{\prod_{j=1}^{k} \operatorname{det} T_{j}} \Xi_{k}^{t}
$$

The choice of the initial conditions for the auxiliary sequences is arbitrary but it is convenient to choose $\Xi_{0}$ in such a way that the first column of $\Xi_{n}$ will contain $\wp_{n}^{\ell}$ and $\wp_{n-1}^{\ell-1}$ and the second column the corresponding second kind polynomials. Since the matrices constructed with the polynomials $\wp_{n_{k}}^{\ell_{k}}$ and the second kind polynomials already satisfy the same recursion relation for $k \geq 1$, it is sufficient to impose the same initial conditions with the following choice (recall that the first move is always a circle-move)

$$
\Xi_{0}=\left(C_{n_{1}}^{\ell_{1}}\right)^{-1}\left[\begin{array}{cc}
\wp_{n_{1}}^{\ell_{1}} & \mathcal{R}_{n_{1}}^{\ell_{1}} \\
\wp_{n_{1}-1}^{\ell_{1}-1} & \mathcal{R}_{n_{1}-1}^{\ell_{1}-1}
\end{array}\right]=\frac{1}{\mu_{0}^{2} x}\left[\begin{array}{cc}
\mu_{0} & \mu_{1} \mu_{0} \\
1 & \mu_{1}-\mu_{0} x
\end{array}\right]\left[\begin{array}{cc}
\mu_{0} x-\mu_{1} & \mu_{0}^{2} \\
1 & 0
\end{array}\right]=\left[\begin{array}{cc}
1 & \frac{\mu_{0}}{x} \\
0 & \frac{1}{x}
\end{array}\right]
$$

Recall that for any $2 \times 2$ matrix we have $A^{t}=\operatorname{det}(A) \mathbf{j} A^{-1} \mathbf{j}^{-1}$. We now compute

$$
\begin{aligned}
\Xi_{k}^{\star}(x) \mathbf{j} \Xi_{k}(y) & =\frac{1}{\operatorname{det} T_{k}(x)} \Xi_{k-1}^{\star}(x) T_{k}^{t}(x) \mathbf{j} T_{k}(y) \Xi_{k-1}(y)= \\
& =\Xi_{k-1}^{\star}(x) \mathbf{j} T_{k}^{-1}(x) T_{k}(y) \Xi_{k-1}(y)= \\
& =\Xi_{k-1}^{\star}(x) \mathbf{j} \Xi_{k-1}(y)+\Xi_{k-1}^{\star}(x)\left(\mathbf{j} T_{k}^{-1}(x) T_{k}(y)-\mathbf{j}\right) \Xi_{k-1}(y)
\end{aligned}
$$

Let us define $\dot{\ell}_{k}:=\ell_{k}-\ell_{k-1}$ and $\dot{n}_{k}:=n_{k}-n_{k-1}$. Then the three formulæ (2-1012-15/2-19) can be uniformly written

$$
\begin{aligned}
& \mathbf{j} T_{k}^{-1}(x) T_{k}(y)-\mathbf{j}=(-1)^{1-\dot{n}_{k}}\left(\frac{1}{y}-\frac{1}{x}\right) x^{\dot{\ell}_{k}} y^{\dot{n}_{k}}\left[\begin{array}{cc}
\frac{\Delta_{n_{k}-1}^{\ell_{k}-1}}{\Delta_{n_{k}}^{\ell_{k}}} & 1-\dot{n}_{k} \\
\dot{\ell}_{k}-1 & 0
\end{array}\right] \\
& \operatorname{det} T_{k}(x)=(-1)^{\dot{n}_{k}} x^{\dot{n}_{k}-\dot{\ell}_{k}}\left(\frac{\Delta_{n_{k}}^{\ell_{k}}}{\Delta_{n_{k-1}}^{\ell_{k-1}}}\right)^{2} \\
& \prod_{j=1}^{k} \operatorname{det} T_{j}(x)=(-1)^{n_{k}} x^{n_{k}-\ell_{k}}\left(\Delta_{n_{k}}^{\ell_{k}}\right)^{2}
\end{aligned}
$$


Summing up both sides of eq. (3-8) we obtain the following master Christoffel-Darboux identity

$$
\begin{aligned}
& \Xi_{N}^{\star}(x) \mathbf{j} \Xi_{N}(y)-\left[\begin{array}{cc}
0 & -1 / y \\
1 / x & 0
\end{array}\right]= \\
& =\left(\frac{1}{y}-\frac{1}{x}\right) \sum_{k=0}^{N-1}(-1)^{1-\dot{n}_{k+1}} x^{\dot{\ell}_{k+1}} y^{\dot{n}_{k+1}} \Xi_{k}^{\star}(x)\left[\begin{array}{cc}
\frac{\Delta_{n_{k+1}-1}^{\ell_{k+1}-1}}{\Delta_{n_{k+1}}^{\ell_{k+1}}} & 1-\dot{n}_{k+1} \\
\dot{\ell}_{k+1}-1 & 0
\end{array}\right] \Xi_{k}(y)= \\
& =\left(\frac{1}{x}-\frac{1}{y}\right) \sum_{k=0}^{N-1}(-1)^{-\dot{n}_{k+1}} x^{\dot{\ell}_{k+1}} y^{\dot{n}_{k+1}} \Xi_{k}^{\star}(x)\left[\begin{array}{cc}
\frac{\Delta_{n_{k+1}-1}^{\ell_{k+1}-1}}{\Delta_{n_{k+1}}^{\ell_{k+1}}} & 1-\dot{n}_{k+1} \\
\dot{\ell}_{k+1}-1 & 0
\end{array}\right] \Xi_{k}(y)
\end{aligned}
$$

\subsection{Principal CDI}

We look at the $(1,1)$ entry of the above identity

$$
\begin{aligned}
& \frac{(-1)^{n_{N}}}{\left(\Delta_{n_{N}}^{\ell_{N}}\right)^{2}}\left(\frac{\wp_{n_{N}-1}^{\ell_{N}-1}(x)}{x^{n_{N}-\ell_{N}}} \wp_{n_{N}}^{\ell_{N}}(y)-\frac{\wp_{n_{N}}^{\ell_{N}}(x)}{x^{n_{N}-\ell_{N}}} \wp_{n_{N}-1}^{\ell_{N}-1}(y)\right)=\left(\frac{1}{x}-\frac{1}{y}\right) \sum_{k=0}^{N-1} \frac{(-1)^{n_{k+1}} x^{\ell_{k+1}-n_{k}} y^{\dot{n}_{k+1}}}{\left(\Delta_{n_{k}}^{\ell_{k}}\right)^{2}} \times \\
& \times\left[\frac{\Delta_{n_{k+1}-1}^{\ell_{k+1}-1}}{\Delta_{n_{k+1}}^{\ell_{k+1}}} \wp_{n_{k}}^{\ell_{k}}(y)+\left(1-\dot{n}_{k+1}\right) \wp_{n_{k}-1}^{\ell_{k}-1}(y)\right]\left[\wp_{n_{k}}^{\ell_{k}}(x)-\left(1-\dot{\ell}_{k+1}\right) \frac{\Delta_{n_{k+1}}^{\ell_{k+1}}}{\Delta_{n_{k+1}-1}^{\ell_{k+1}-1}} \wp_{n_{k}-1}^{\ell_{k}-1}(x)\right]
\end{aligned}
$$

The two terms in the product inside the sum here above can be simplified using (2-7) for the case $\dot{\ell}_{k+1}=0$ and (2-9) for the case $\dot{n}_{k+1}=0$ indeed

$$
\begin{aligned}
& {\left[\frac{\Delta_{n_{k+1}-1}^{\ell_{k+1}-1}}{\Delta_{n_{k+1}}^{\ell_{k+1}}} \wp_{n_{k}}^{\ell_{k}}(y)+\left(1-\dot{n}_{k+1}\right) \wp_{n_{k}-1}^{\ell_{k}-1}(y)\right]= \begin{cases}y \frac{\Delta_{n_{k}}^{\ell_{k}}}{\Delta_{n_{k}}^{\ell_{k}+1}} \wp_{n_{k+1}-1}^{\ell_{k}}(y) & \text { if } \dot{n}_{k+1}=0 \\
\frac{\Delta_{n_{k+1}-1}^{\ell_{k+1}-1}}{\Delta_{n_{k+1}}^{\ell_{k+1}}} \wp_{n_{k}}^{\ell_{k}}(y) & \text { if } \dot{n}_{k+1}=1\end{cases} } \\
& =y^{1-\dot{n}_{k+1}} \frac{\Delta_{n_{k}}^{\ell_{k+1}-1}}{\Delta_{n_{k+1}}^{\ell_{k+1}}} \wp_{n_{k+1}-1}^{\ell_{k}}(y) \\
& {\left[\wp_{n_{k}}^{\ell_{k}}(x)-\left(1-\dot{\ell}_{k+1}\right) \frac{\Delta_{n_{k+1}}^{\ell_{k+1}}}{\Delta_{n_{k+1}-1}^{\ell_{k+1}-1}} \wp_{n_{k}-1}^{\ell_{k}-1}(x)\right]= \begin{cases}\frac{\Delta_{n_{k}}^{\ell_{k+1}}}{\Delta_{n_{k+1}-1}^{\ell_{k+1}-1}} \wp_{n_{k}}^{\ell_{k+1}-1}(x) & \text { if } \dot{\ell}_{k+1}=0 \\
\wp_{n_{k}}^{\ell_{k}}(x)=\wp_{n_{k}}^{\ell_{k+1}-1}(x) & \text { if } \dot{\ell}_{k+1}=1\end{cases} } \\
& =\frac{\Delta_{n_{k}}^{\ell_{k}}}{\Delta_{n_{k}}^{\ell_{k+1}-1}} \wp_{n_{k}}^{\ell_{k+1}-1}(x)
\end{aligned}
$$

Using these expression in the RHS of (3-13) the identity becomes

$$
\begin{aligned}
& \frac{(-1)^{n_{N}}}{\left(\Delta_{n_{N}}^{\ell_{N}}\right)^{2}}\left(\frac{\wp_{n_{N}-1}^{\ell_{N}-1}(x)}{x^{n_{N}-\ell_{N}}} \wp_{n_{N}}^{\ell_{N}}(y)-\frac{\wp_{n_{N}}^{\ell_{N}}(x)}{x^{n_{N}-\ell_{N}}} \wp_{n_{N}-1}^{\ell_{N}-1}(y)\right)= \\
= & \left(\frac{y}{x}-1\right) \sum_{k=0}^{N-1}(-1)^{n_{k+1}} \frac{\wp_{n_{k+1}-1}^{\ell_{k}}(y) \wp_{n_{k}}^{\ell_{k+1}-1}(x) x^{\ell_{k+1}-n_{k}}}{\Delta_{n_{k+1}}^{\ell_{k+1}} \Delta_{n_{k}}^{\ell_{k}}}
\end{aligned}
$$


We can repeat the same arguments for the second-kind polynomials appearing in the other matrix entries; care must be paid to the fact that $\left(\Xi_{0}\right)_{12}$ is not $\mathcal{R}_{0}^{0} \equiv 0$.

We obtain the following supplementary CDI's (provided that $0 \leq \ell_{k} \leq n_{k+1}-2, k=1, \ldots$ )

$$
\begin{aligned}
& \frac{(-1)^{n_{N}}}{\left(\Delta_{n_{N}}^{\ell_{N}}\right)^{2}}\left(\frac{\mathcal{R}_{n_{N}-1}^{\ell_{N}-1}(x)}{x^{n_{N}-\ell_{N}}} \wp_{n_{N}}^{\ell_{N}}(y)-\frac{\mathcal{R}_{n_{N}}^{\ell_{N}}(x)}{x^{n_{N}-\ell_{N}}} \wp_{n_{N}-1}^{\ell_{N}-1}(y)\right)-\frac{1}{x}= \\
& =\left(\frac{y}{x}-1\right) \sum_{k=0}^{N-1}(-1)^{n_{k+1}} \frac{\wp_{n_{k+1}-1}^{\ell_{k}}(y) \mathcal{R}_{n_{k}}^{\ell_{k+1}-1}(x) x^{\ell_{k+1}-n_{k}}}{\Delta_{n_{k+1}}^{\ell_{k}} \Delta_{n_{k}}^{\ell_{k}}} \\
& \frac{(-1)^{n_{N}}}{\left(\Delta_{n_{N}}^{\ell_{N}}\right)^{2}}\left(\frac{\wp_{n_{N}-1}^{\ell_{N}-1}(x)}{x^{n_{N}-\ell_{N}}} \mathcal{R}_{n_{N}}^{\ell_{N}}(y)-\frac{\wp_{n_{N}}^{\ell_{N}}(x)}{x^{n_{N}-\ell_{N}}} \mathcal{R}_{n_{N}-1}^{\ell_{N}-1}(y)\right)+\frac{1}{y}= \\
& =\left(\frac{y}{x}-1\right)\left[\sum_{k=0}^{N-1}(-1)^{n_{k+1}} \frac{\mathcal{R}_{n_{k+1}-1}^{\ell_{k}}(y) \wp_{n_{k}}^{\ell_{k+1}-1}(x) x^{\ell_{k+1}-n_{k}}}{\Delta_{n_{k+1}}^{\ell_{k+1}} \Delta_{n_{k}}^{\ell_{k}}}\right. \\
& \frac{(-1)^{n_{N}}}{\left(\Delta_{n_{N}}^{\ell_{N}}\right)^{2}}\left(\frac{\mathcal{R}_{n_{N}-1}^{\ell_{N}-1}(x)}{x^{n_{N}-\ell_{N}}} \mathcal{R}_{n_{N}}^{\ell_{N}}(y)-\frac{\mathcal{R}_{n_{N}}^{\ell_{N}}(x)}{x^{n_{N}-\ell_{N}}} \mathcal{R}_{n_{N}-1}^{\ell_{N}-1}(y)\right)= \\
& =\left(\frac{y}{x}-1\right) \sum_{k=0}^{N-1}(-1)^{n_{k+1}} \frac{\mathcal{R}_{n_{k+1}-1}^{\ell_{k}}(y) \mathcal{R}_{n_{k}}^{\ell_{k+1}-1}(x) x^{\ell_{k+1}-n_{k}}}{\Delta_{n_{k+1}}^{\ell_{k+1}} \Delta_{n_{k}}^{\ell_{k}}}
\end{aligned}
$$

The additional term in the second identity stems from the mentioned discrepancy in the definition of $\Xi_{0}$ with the definition of the auxiliary polynomials: indeed the term with $k=0$ in the sum (3-12) is not zero in the off-diagonal terms but $\left[\begin{array}{cc}1 & -\frac{1}{y} \\ 0 & 0\end{array}\right]$. Thus the second identity above is rewritten as

$$
\begin{array}{r}
\frac{(-1)^{n_{N}}}{\left(\Delta_{n_{N}}^{\ell_{N}}\right)^{2}}\left(\frac{\wp_{n_{N}-1}^{\ell_{N}-1}(x)}{x^{n_{N}-\ell_{N}}} \mathcal{R}_{n_{N}}^{\ell_{N}}(y)-\frac{\wp_{n_{N}}^{\ell_{N}}(x)}{x^{n_{N}-\ell_{N}}} \mathcal{R}_{n_{N}-1}^{\ell_{N}-1}(y)\right)+\frac{1}{x}= \\
=\left(\frac{y}{x}-1\right) \sum_{k=0}^{N-1}(-1)^{n_{k+1}} \frac{\mathcal{R}_{n_{k+1}-1}^{\ell_{k}}(y) \wp_{n_{k}}^{\ell_{k+1}-1}(x) x^{\ell_{k+1}-n_{k}}}{\Delta_{n_{k+1}}^{\ell_{k+1}} \Delta_{n_{k}}^{\ell_{k}}}
\end{array}
$$

\section{CDIs for biorthogonal Laurent polynomials}

The formulæ derived in the previous sections for the Christoffel-Darboux identities are very general however the (Laurent) polynomials that appear in the sum are not biorthogonal with respect to the moment functional $\mathcal{L}$ unless the sequence $n_{k}$ is strictly increasing and the sequence $\ell_{k}$ is weakly increasing. This is the situation which interests us the most and hence from now on we will assume that $n_{k}=k^{5}$. Moreover all the elementary orbits of the integrable lattices we are considering are in correspondence with this situation.

From the formulæ defining the polynomials $\wp_{n}^{\ell}$ it follows that

$$
\mathcal{L}_{z}\left(\wp_{n}^{\ell_{n}}(z) \wp_{m}^{\ell_{m+1}-1}(z) z^{\ell_{m+1}-m}\right)=\delta_{m n}(-1)^{n} \Delta_{n}^{\ell_{n}} \Delta_{n+1}^{\ell_{n+1}} .
$$

This suggests that we introduce the following monic polynomials

$$
\pi_{n}(x)=\frac{1}{\Delta_{n}^{\ell_{n}}} \wp_{n}^{\ell_{n}}(x)
$$

\footnotetext{
${ }^{5}$ If $n_{k}$ were not strictly increasing then the polynomials would be biorthogonal only provided the moments satisfy some non generic condition of vanishing of certain determinants.
} 


$$
\rho_{n}(x)=\frac{(-1)^{n}}{\Delta_{n}^{\ell_{n}}} x^{\ell_{n+1}-n} \wp_{n}^{\ell_{n+1}-1}(x) .
$$

It is understood that the determinants $\Delta_{n}^{\ell_{n}}$ must not vanish: this is our implicit assumption of genericity on the moment functional. While the $\pi_{n}$ 's are monic in the usual sense, the $\rho_{n}$ 's are normalized on either the highest or the lowest power depending on $\dot{\ell}_{n+1}$. Moreover the $\pi_{n}$ 's are polynomials in $x$ whereas the $\rho_{n}$ 's are polynomials in $x$ and $x^{-1}$. They satisfy the orthogonality relations

$$
\mathcal{L}_{z}\left(\rho_{m}(z) \pi_{n}(z)\right)=\delta_{m n} h_{n}, \quad h_{n}:=\frac{\Delta_{n+1}^{\ell_{n+1}}}{\Delta_{n}^{\ell_{n}}}
$$

We finally introduce the (bi)-orthonormal polynomials and the second kind polynomials

$$
\begin{aligned}
& p_{n}(x):=\frac{1}{\sqrt{h_{n}}} \pi_{n}(x)=\frac{\wp_{n}^{\ell_{n}}}{\sqrt{\Delta_{n}^{\ell_{n}} \Delta_{n+1}^{\ell_{n}+1}}}, \\
& \widetilde{p}_{n}(x):=\mathcal{L}_{z}\left(\frac{p_{n}(x)-p_{n}(z)}{x-z}\right) \\
& r_{n}(x):=\frac{1}{\sqrt{h_{n}}} \rho_{n}(x)=x^{\ell_{n+1}-n} \frac{(-1)^{n} \wp_{n}^{\ell_{n}+1}-1}{\sqrt{\Delta_{n}^{\ell_{n}} \Delta_{n+1}^{\ell_{n+1}}}} \\
& \widetilde{r}_{n}(x):=\mathcal{L}_{z}\left(\frac{r_{n}(x)-r_{n}(z)}{x-z}\right)
\end{aligned}
$$

and their "starred"

$$
\begin{array}{rr}
p_{n}^{\star}(x):=x^{\ell_{n}-n+1} p_{n}(x) & \widetilde{p}_{n}^{\star}(x):=x^{\ell_{n}-n+1} \widetilde{p}_{n}(x) \\
r_{n}^{\star}(x):=x^{n-\ell_{n+1}} r_{n}(x) & \widetilde{r}_{n}^{\star}(x):=x^{n-\ell_{n+1}} \widetilde{r}_{n}(x)
\end{array}
$$

In terms of these (Laurent)polynomials the CDIs read

$$
\begin{aligned}
(y-x) \sum_{n=0}^{N-1} r_{n}(x) p_{n}(y)= & \gamma_{N}\left(p_{N}(y) r_{N-1}(x)-p_{N}^{\star}(x) r_{N-1}^{\star}(y)\right) \\
(y-x) \sum_{n=0}^{N-1} \widetilde{r}_{n}(x) p_{n}(y)= & \gamma_{N}\left(p_{N}(y) \widetilde{r}_{N-1}(x)-\widetilde{p}_{N}^{\star}(x) r_{N-1}^{\star}(y)\right)+1 \\
(y-x) \sum_{n=0}^{N-1} r_{n}(x) \widetilde{p}_{n}(y)= & \gamma_{N}\left(\widetilde{p}_{N}(y) r_{N-1}(x)-p_{N}^{\star}(x) \widetilde{r}_{N-1}^{\star}(y)\right)-1 \\
(y-x) \sum_{n=0}^{N-1} \widetilde{r}_{n}(x) \widetilde{p}_{n}(y)= & \gamma_{N}\left(\widetilde{p}_{N}(y) \widetilde{r}_{N-1}(x)-\widetilde{p}_{N}^{\star}(x) \widetilde{r}_{N-1}^{\star}(y)\right) \\
& \gamma_{N}:=\sqrt{\frac{h_{N}}{h_{N-1}}}
\end{aligned}
$$

It is convenient to rewrite in matrix form the previous identities as follows

$$
\begin{aligned}
& \mathbf{p}(x):=\left[p_{0}, \ldots\right]^{t}, \widetilde{\mathbf{p}}(x):=\left[\widetilde{p}_{0}, \ldots\right]^{t}, \mathbf{r}(x):=\left[r_{0}, \cdots\right]^{t}, \widetilde{\mathbf{r}}(x):=\left[\widetilde{r}_{0}, \cdots\right]^{t} \\
& \mathbf{P}(x):=[\mathbf{p}(x), \widetilde{\mathbf{p}}(x)], \quad \mathbf{R}(x):=[\mathbf{r}(x), \widetilde{\mathbf{r}}(x)]
\end{aligned}
$$




$$
\begin{aligned}
& \left(\Pi_{N-1}\right)_{i j}:=\sum_{k=0}^{N-1} \delta_{i k} \delta_{k j} \\
& \mathbf{R}^{t}(x) \Pi_{N-1} \mathbf{P}(y)=\frac{1}{y-x}\left\{\gamma_{N}\left[\begin{array}{cc}
p_{N}^{\star}(x) & r_{N-1}(x) \\
\widetilde{p}_{N}^{\star}(x) & \widetilde{r}_{N-1}(x)
\end{array}\right] \mathbf{j}\left[\begin{array}{cc}
p_{N}(y) & \widetilde{p}_{N}(y) \\
r_{N-1}^{\star}(y) & \widetilde{r}_{N-1}^{\star}(y)
\end{array}\right]+\mathbf{j}\right\}
\end{aligned}
$$

Remark 4.1 A word about the relations with previously known (bi)-orthogonal polynomials is now in order. If all the moves (except the first one) are line-moves namely if $\ell_{n}=n-1$ then it is not hard to show that $\pi_{n}=\rho_{n}$ are just orthogonal polynomials with respect to the (restriction of the) moment functional $\mathcal{L}$ to positive moments. Moreover the shifted Töplitz determinants $\Delta_{n}^{n-1}$ are (up to a sign) the same as the Hänkel determinants of the same size (by permuting appropriately the columns).

Vice-versa, if all moves are circle-moves (i.e. $\ell_{n} \equiv 0$ ) (and we also impose certain reality conditions on the moments of the functional) then the $\pi_{n}$ are orthogonal polynomials for a certain measure on the unit circle and the $\rho_{n}$ 's are their so-called "dual" Laurent polynomials. The determinants appearing then in our sequence are precisely the "standar" ones $\Delta_{n}^{0}$.

A second remark is that all these polynomial do satisfy three-terms recurrence relations, although of a different sort than the standard ones. Indeed, it is well known that orthogonal polynomials $p_{n}$ satisfy relations of the form

$$
x p_{n}=\gamma_{n} p_{n+1}+\beta_{n} p_{n}+\gamma_{n-1} p_{n-1},
$$

where the coefficients $\gamma_{n}, \beta_{n}$ enter in the tridiagonal Jacobi matrix representing the multiplication by $x$ in the basis of the $p_{n}$ 's. At the opposite "end of the spectrum", orthogonal polynomials on the circle satisfy a different sort of three term recurrence relation, of the form

$$
x\left(p_{n}+\delta_{n} p_{n-1}\right)=\gamma_{n} p_{n+1}+\beta_{n} p_{n} .
$$

It is not hard to show [9, 10] that the polynomials that we are considering precisely "interpolate" these two sorts of recurrence relations as follows

$$
x\left(p_{n}+\left(1-\dot{\ell}_{n}\right) \delta_{n} p_{n-1}\right)=\gamma_{n} p_{n+1}+\dot{\ell}_{n} \beta_{n} p_{n},
$$

for certain coefficients $\gamma_{n}, \beta_{n}, \delta_{n}$ whose explicit expression in terms of Töplitz determinants can be obtained from the formula above but is irrelevant for this discussion. We see that "circle moves" $\left(\dot{\ell}_{n}=0\right)$ correspond to a three-term recurrence relation of the type appearing for O.P. on the circle, while "line moves" $\left(\dot{\ell}_{n}=1\right)$ correspond to the "usual" recurrence relation.

\section{Infinitesimal deformations of the moment functional}

We study the infinitesimal deformations for the wave vectors $\mathbf{p}(x), \widetilde{\mathbf{p}}(x), \mathbf{r}(x)$ and $\widetilde{\mathbf{r}}(x)$ under an infinitesimal deformation of the moment functional. Let us introduce the matrix of recurrence for these sequences of polynomials

$$
x \mathbf{p}=Q \mathbf{p} ; x \mathbf{r}^{t}=\mathbf{r}^{t} Q, \quad Q_{n m}:=\mathcal{L}\left(z p_{n} r_{m}\right) .
$$

The matrix $Q$ is of Hessenberg form, namely has nonzero entries on the superdiagonal and possibly on the diagonal and all other nonzero entries in the lower triangular part. The biorthogonality relation can be rewritten as

$$
\mathcal{L}\left[\mathbf{p r}^{t}\right]=\mathbf{1}
$$


Suppose we infinitesimally deform the moment functional

$$
\dot{\mathcal{L}}(\bullet)=-\mathcal{L}(F(z) \bullet)
$$

Here $F(z)$ can be any function (even a generalized distribution as we will see) provided that the moments of the deformation are still well defined: if $\mathcal{L}$ is given by an analytical expression in terms of some integral representation (as we will assume later on) then this means some condition of analyticity on $F$ : if the functional is only defined by its moments, then $F$ should be interpreted as formal series. In any situation the typical case of $F$ being a polynomial (corresponding to the usual formal Toda-type flows) will be well defined.

A little more generally we could even assume that $F$ is a distribution, particularly delta functions or derivatives of it. For instance we can consider deformation of the type

$$
\delta \mathcal{L}(p(x)) \equiv \dot{\mathcal{L}}(p(x))=-\left.\left(\frac{\mathrm{d}}{\mathrm{d} x}\right)^{k} p(x)\right|_{x=a}
$$

for some constant $a$ : this means that we (formally) have set $F$ to be the $k$-th derivative of the Dirac delta distribution for the given moment functional supported at $x=a$.

Corresponding to any of these deformations the BOPs deform as

$$
\delta \mathbf{p}=\mathbb{U}^{(F)} \mathbf{p}, \quad \delta \mathbf{r}=\widetilde{\mathbb{U}}^{(F)} \mathbf{r}
$$

where a priori $\mathbb{U}$ and $\widetilde{\mathbb{U}}$ are lower triangular matrices since the range of powers of $x$ entering in the expressions $p_{n}, r_{n}$ will not change. In order to find expressions for these matrices we note first that their diagonals are the same

$$
\left(\mathbb{U}^{(F)}\right)_{n n}=\left(\widetilde{\mathbb{U}}^{(F)}\right)_{n n}=-\frac{1}{2} \delta \ln \left(h_{n}\right)
$$

Indeed we have

$$
\begin{aligned}
\delta p_{n}=\delta \frac{x^{n}}{\sqrt{h_{n}}}+\ldots & =-\frac{1}{2} \delta \ln \left(h_{n}\right) p_{n}+\text { previous } \\
\delta r_{n} & =-\frac{1}{2} \delta \ln \left(h_{n}\right) r_{n}+\text { previous }
\end{aligned}
$$

Differentiating the orthogonality relation we obtain

$$
\mathbb{U}^{(F)}+\widetilde{\mathbb{U}}^{(F) t}=\left\{\begin{array}{cc}
F(Q) & \text { for the case of an ordinary function } F \\
\left.\left(\frac{\mathrm{d}}{\mathrm{d} x}\right)^{k} \mathbf{p}(x) \mathbf{r}^{t}(x)\right|_{x=a} & \text { for a deformation supported at one point }
\end{array}\right.
$$

and hence according to the two types the matrices describing the infinitesimal deformations are given by

$$
\begin{array}{cl}
\mathbb{U}^{(F)}=F(Q)_{-0}, & \widetilde{\mathbb{U}}^{(F)}=F(Q)^{t}{ }_{-0} \\
\mathbb{U}^{\left(\delta_{a}^{k}\right)}=\partial_{a}^{k}\left(\mathbf{p}(a) \mathbf{r}^{t}(a)\right)_{-0}, & \widetilde{\mathbb{U}}^{\left(\delta_{a}^{k}\right)}=\partial_{a}^{k}\left(\mathbf{r}(a) \mathbf{p}^{t}(a)\right)_{-0}
\end{array}
$$

where $A_{-0}$ means the lower triangular part plus half of the diagonal. Note that from (5-6) and the definition of $h_{n}$ it follows

$$
\Delta_{n}^{\ell_{n}}=\prod_{k=0}^{n-1} h_{k}
$$




$$
\begin{aligned}
& \delta_{f} \ln \Delta_{n}^{\ell_{n}}=-\operatorname{Tr}_{n} F(Q) \\
& \delta_{\delta_{a}^{k}} \ln \Delta_{n}^{\ell_{n}}=-\partial_{a}^{k} \sum_{j=0}^{n-1} p_{j}(a) r_{j}(a),
\end{aligned}
$$

where we have used the notation for the truncated $\operatorname{trace} \operatorname{Tr}_{n} A:=\sum_{j=0}^{n-1} A_{j j}$.

\subsection{Deformations for the second-kind (Laurent) polynomials}

Using Leibnitz's rule we obtain the following deformation equations for the second-kind wave vectors $\widetilde{\mathbf{p}}$, $\widetilde{\mathbf{r}}$. For a deformation by a function $F(x)$ we have

$$
\begin{aligned}
& \delta_{F} \widetilde{\mathbf{p}}=\left(\mathbb{U}^{(F)}-F(x)\right) \widetilde{\mathbf{p}}+\mathcal{L}_{z}\left(\frac{F(x)-F(z)}{x-z}\right) \mathbf{p}-\left(\frac{F(x)-F(Q)}{x-Q}\right) \mathbf{e}_{1} \\
& \delta_{F} \widetilde{\mathbf{r}}=\left(\widetilde{\mathbb{U}}^{(F)}-F(x)\right) \widetilde{\mathbf{r}}+\mathcal{L}_{z}\left(\frac{F(x)-F(z)}{x-z}\right) \mathbf{r}-\left(\frac{F(x)-F\left(Q^{t}\right)}{x-Q^{t}}\right) \mathbf{e}_{1}
\end{aligned}
$$

while for $F=\delta_{\mathcal{L}}^{(k)}(z-a)$ we have

$$
\begin{aligned}
& \delta_{F} \widetilde{\mathbf{p}}=\mathbb{U}^{\left(\delta_{a}^{k}\right)} \widetilde{p}-\frac{\partial^{k}}{\partial a^{k}} \frac{\mathbf{p}(x)-\mathbf{p}(a)}{x-a} \\
& \delta_{F} \widetilde{\mathbf{r}}=\widetilde{\mathbb{U}}^{\left(\delta_{a}^{k}\right)} \widetilde{r}-\frac{\partial^{k}}{\partial a^{k}} \frac{\mathbf{r}(x)-\mathbf{r}(a)}{x-a}
\end{aligned}
$$

\section{Folded version of the deformation equations}

Let us define

$$
\chi_{n}:=\left[\begin{array}{cc}
p_{n} & \widetilde{p}_{n} \\
r_{n-1}^{\star} & \widetilde{r}_{n-1}^{\star}
\end{array}\right]
$$

We want to express the previous infinite-dimensional deformation equations in terms of $\chi_{n}$ alone; this process is conceptually identical to the one followed in [4] and which is named "folding". To this end we formulate the following

Theorem 6.1 The infinite deformations (5-10) for the wave vectors $\mathbf{p}, \mathbf{r}$ and for the second-kind wave vectors $\widetilde{\mathbf{p}}, \widetilde{\mathbf{r}}$ [5-13, 5-14) are equivalent to the following deformation equations for $\chi_{n}, n \geq 1$.

$$
\begin{aligned}
\delta_{(F)} \chi_{n} & =\mathcal{U}_{n}^{(F)}(x) \chi_{n}+\chi_{n} \mathcal{U}^{(F), R}(x) \\
\delta_{\left(\delta_{a}^{k}\right)} \chi_{n}(a) & =\mathcal{U}_{n}^{\left(\delta_{a}^{k}\right)}(x) \chi_{n}(x)+\chi_{n}(x) \mathcal{U}^{\left(\delta_{a}^{k}\right), R}(x)
\end{aligned}
$$

where we have used the following definitions:

$$
\begin{aligned}
\mathcal{U}_{n}^{(F)} & =\left[\begin{array}{cc}
\frac{1}{2} F(Q)_{n n} & 0 \\
0 & F(x)-\frac{1}{2} F(Q)_{n-1, n-1}
\end{array}\right]+\gamma_{n}\left[\begin{array}{cc}
-\left(\nabla_{Q} F\right)_{n, n-1} & \left(\nabla_{Q} F\right)_{n, n^{\star}} \\
-\left(\nabla_{Q} F\right)_{(n-1)^{\star}, n-1} & \left(\nabla_{Q} F\right)_{n, n-1}
\end{array}\right] \\
\mathcal{U}^{(F), R} & =\left[\begin{array}{cc}
0 & \mathcal{W}_{F} \\
0 & -F(x)
\end{array}\right], \mathcal{W}_{F}:=\mathcal{L}_{z}\left(\frac{F(x)-F(z)}{x-z}\right), \quad \nabla_{Q} F:=\frac{F(x)-F(Q)}{x-Q} \\
\mathcal{U}_{n}^{\left(\delta_{a}^{k}\right)}(x) & =\frac{\partial^{k}}{\partial a^{k}} \frac{1}{2}\left[\begin{array}{cc}
p_{n} r_{n} & 0 \\
0 & -p_{n-1} r_{n-1}
\end{array}\right]_{z=a}+\frac{\partial^{k}}{\partial a^{k}} \frac{\gamma_{n}}{x-a}\left[\begin{array}{cc}
-p_{n} r_{n-1} & p_{n} p_{n}^{\star} \\
-r_{n-1} r_{n-1}^{\star} & r_{n-1} p_{n}
\end{array}\right]_{z=a}
\end{aligned}
$$




$$
\mathcal{U}^{\left(\delta_{a}^{k}\right), R}(x)=\partial_{a}^{k}\left[\begin{array}{cc}
0 & \frac{1}{a-x} \\
0 & 0
\end{array}\right] .
$$

Here, for a function $f(z)$ we have set

$$
f(Q)_{i, j^{\star}}:=\mathcal{L}\left(r_{i} f(z) r_{i}^{\star}\right), \quad f(Q)_{i^{\star}, j}:=\mathcal{L}\left(p_{i}^{\star} f(z) p_{j}\right) .
$$

Proof. We compute the deformations of both rows of $\chi_{n}$. We start with deformation involving a function $F(x)$ : the first row deforms according to the equation

$$
\delta_{F}\left[p_{n}(x), \widetilde{p}_{n}(x)\right]=\delta_{F} \mathbf{e}_{n}^{t} \cdot[\mathbf{p}, \widetilde{\mathbf{p}}]=\mathbf{e}_{n}^{t} \cdot \mathbb{U}^{(F)} \cdot[\mathbf{p}, \widetilde{\mathbf{p}}]+\mathbf{e}_{n}^{t} \cdot[\mathbf{p} \cdot \widetilde{\mathbf{p}}]\left[\begin{array}{cc}
0 & \mathcal{W}_{F} \\
0 & -F(x)
\end{array}\right]-\mathbf{e}_{n}^{t} \cdot \frac{F(x)-F(Q)}{x-Q} \cdot\left[\mathbf{0}, \mathbf{e}_{1}\right]
$$

where we have set

$$
\mathcal{W}_{F}(x):=\mathcal{L}_{z}\left(\frac{F(x)-F(z)}{x-z}\right)
$$

We now note that

$$
\begin{array}{r}
\mathbf{e}_{n}^{t} \cdot \mathbb{U}^{(F)} \cdot[\mathbf{p}, \widetilde{\mathbf{p}}]=\frac{1}{2} F(Q)_{n n}\left[p_{n}, \widetilde{p}_{n}\right]+\mathbf{e}_{n}^{t} \mathcal{L}_{z}\left(F(z) \mathbf{p}(z) \mathbf{r}^{t}(z) \Pi_{n-1}[\mathbf{p}(x), \widetilde{\mathbf{p}}(x)]\right)= \\
=\frac{1}{2} F(Q)_{n n}\left[p_{n}, \widetilde{p}_{n}\right]+\mathbf{e}_{n}^{t} \mathcal{L}_{z}\left((F(z)-F(x)) \mathbf{p}(z) \mathbf{r}^{t}(z) \Pi_{n-1}[\mathbf{p}(x), \widetilde{\mathbf{p}}(x)]\right)= \\
=\frac{1}{2} F(Q)_{n n}\left[p_{n}, \widetilde{p}_{n}\right]+\mathbf{e}_{n}^{t} \mathcal{L}_{z}\left(\frac{F(z)-F(x)}{x-z} \mathbf{p}(z)\left(\gamma_{n}\left[p_{n}(z)^{\star}, r_{n-1}(z)\right] \mathbf{j} \chi_{n}(x)-[0,1]\right)\right)= \\
=\frac{1}{2} F(Q)_{n n}\left[p_{n}, \widetilde{p}_{n}\right]-\gamma_{n} \mathcal{L}_{z}\left(\frac{F(z)-F(x)}{z-x} p_{n}\left[p_{n}^{\star}, r_{n-1}\right]\right) \mathbf{j} \chi_{n}(x)+\mathbf{e}_{n}^{t} \cdot \frac{F(x)-F(Q)}{x-Q} \cdot\left[\mathbf{0}, \mathbf{e}_{1}\right]
\end{array}
$$

This implies that

$$
\begin{array}{r}
\delta_{F}\left[p_{n}(x), \widetilde{p}_{n}(x)\right]=\frac{1}{2} F(Q)_{n n}\left[p_{n}, \widetilde{p}_{n}\right]-\gamma_{n} \mathcal{L}_{z}\left(\frac{F(z)-F(x)}{z-x} p_{n}\left[p_{n}^{\star}, r_{n-1}\right]\right) \mathbf{j} \chi_{n}(x)+ \\
+\mathbf{e}_{n}^{t} \cdot[\mathbf{p} \cdot \widetilde{\mathbf{p}}]\left[\begin{array}{cc}
0 & \mathcal{W}_{F} \\
0 & -F(x)
\end{array}\right]
\end{array}
$$

In a similar way we can compute the following deformations

$$
\begin{aligned}
& \delta_{F}\left[r_{n-1}(x), \widetilde{r}_{n-1}(x)\right]=\delta_{F} \mathbf{e}_{n-1}^{t} \cdot[\mathbf{r}, \widetilde{\mathbf{r}}]= \\
& =\mathbf{e}_{n-1}^{t} \cdot \mathbb{U}^{(F) t} \cdot[\mathbf{r}, \widetilde{\mathbf{r}}]+\mathbf{e}_{n-1}^{t} \cdot[\mathbf{r}, \widetilde{\mathbf{r}}]\left[\begin{array}{cc}
0 & \mathcal{W}_{F} \\
0 & -F(x)
\end{array}\right]-\mathbf{e}_{n-1}^{t} \cdot \frac{F(x)-F\left(Q^{t}\right)}{x-Q^{t}} \cdot\left[\mathbf{0}, \mathbf{e}_{1}\right] .
\end{aligned}
$$

The computation now involves

$$
\begin{aligned}
\mathbf{e}_{n-1}^{t} \cdot \mathbb{U}^{(F) t} \cdot[\mathbf{r}, \widetilde{\mathbf{r}}]= & -\frac{1}{2} F(Q)_{n-1, n-1}\left[r_{n-1}, \widetilde{r}_{n-1}\right]+\mathbf{e}_{n-1}^{t} \cdot F\left(Q^{t}\right) \Pi_{n-1}[\mathbf{r}, \widetilde{\mathbf{r}}]= \\
= & -\frac{1}{2} F(Q)_{n-1, n-1}\left[r_{n-1}, \widetilde{r}_{n-1}\right]+\mathbf{e}_{n-1}^{t} \mathcal{L}_{z}\left(F(z) \mathbf{r}(z) \mathbf{p}^{t}(z) \Pi_{n-1}[\mathbf{r}(x), \widetilde{\mathbf{r}}(x)]\right)= \\
= & \left(F(x)-\frac{1}{2} F(Q)_{n-1, n-1}\right)\left[r_{n-1}, \widetilde{r}_{n-1}\right]+ \\
& +\mathbf{e}_{n-1}^{t} \mathcal{L}_{z}\left((F(z)-F(x)) \mathbf{r}(z) \mathbf{p}^{t}(z) \Pi_{n-1}[\mathbf{r}(x), \widetilde{\mathbf{r}}(x)]\right)= \\
= & \left(F(x)-\frac{1}{2} F(Q)_{n-1, n-1}\right)\left[r_{n-1}, \widetilde{r}_{n-1}\right]+
\end{aligned}
$$




$$
\begin{aligned}
& +\mathbf{e}_{n-1} \mathcal{L}_{z}\left(\frac{F(z)-F(x)}{z-x} \mathbf{r}(z)\left(-\gamma_{n}\left[p_{n}(z), r_{n-1}^{\star}(z)\right] \mathbf{j} \chi_{n}^{\star}(x)^{t}+[0,1]\right)\right)= \\
= & \left(F(x)-\frac{1}{2} F(Q)_{n-1, n-1}\right)\left[r_{n-1}, \widetilde{r}_{n-1}\right]+ \\
& -\gamma_{n} \mathcal{L}_{z}\left(\frac{F(z)-F(x)}{z-x} r_{n-1}\left[p_{n}, r_{n-1}^{\star}\right]\right) \mathbf{j} \chi_{n}^{\star t}(x)+\mathbf{e}_{n-1} \frac{F(x)-F\left(Q^{t}\right)}{x-Q^{t}} \mathbf{e}_{1}
\end{aligned}
$$

where we have used the following definition

$$
\chi_{n}^{\star}=x^{\ell_{n}-n+1} \chi_{n}^{t} .
$$

Thus we have obtained the following deformation equation

$$
\begin{aligned}
\delta_{F}\left[r_{n-1}(x), \widetilde{r}_{n-1}(x)\right]= & \left(F(x)-\frac{1}{2} F(Q)_{n-1, n-1}\right)\left[r_{n-1}, \widetilde{r}_{n-1}\right]+\mathbf{e}_{n-1}^{t} \cdot[\mathbf{r}, \widetilde{\mathbf{r}}]\left[\begin{array}{cc}
0 & \mathcal{W}_{F} \\
0 & -F(x)
\end{array}\right]+ \\
& -\gamma_{n} \mathcal{L}_{z}\left(\frac{F(z)-F(x)}{z-x} r_{n-1}\left[p_{n}, r_{n-1}^{\star}\right]\right) \mathbf{j} \chi_{n}^{\star t}(x)
\end{aligned}
$$

By "starifying" both sides we obtain

$$
\begin{aligned}
\delta_{F}\left[r_{n-1}^{\star}(x), \widetilde{r}_{n-1}^{\star}(x)\right]= & \left(F(x)-\frac{1}{2} F(Q)_{n-1, n-1}\right)\left[r_{n-1}^{\star}, \widetilde{r}_{n-1}^{\star}\right]+\left[r_{n-1}^{\star}, \widetilde{r}_{n-1}^{\star}\right]\left[\begin{array}{cc}
0 & \mathcal{W}_{F} \\
0 & -F(x)
\end{array}\right]+ \\
& -\gamma_{n} \mathcal{L}_{z}\left(\frac{F(z)-F(x)}{z-x} r_{n-1}\left[p_{n}, r_{n-1}^{\star}\right]\right) \mathbf{j} \chi_{n}(x)
\end{aligned}
$$

Putting together (6-9) and (6-14) we obtain finally

$$
\begin{aligned}
& \delta_{F} \chi_{n}=\mathcal{U}_{n}^{(F)}(x) \chi_{n}+\chi_{n} \mathcal{U}^{(F), R}(x) \\
& \mathcal{U}_{n}^{(F)}=\left[\begin{array}{cc}
\frac{1}{2} F(Q)_{n n} & 0 \\
0 & F(x)-\frac{1}{2} F(Q)_{n-1, n-1}
\end{array}\right]-\gamma_{n} \mathcal{L}_{z}\left(\frac{F(x)-F(z)}{x-z}\left[\begin{array}{cc}
p_{n} p_{n}^{\star} & p_{n} r_{n-1} \\
p_{n} r_{n-1} & r_{n-1}^{\star} r_{n-1}
\end{array}\right]\right) \mathbf{j}= \\
& =\left[\begin{array}{cc}
\frac{1}{2} F(Q)_{n n} & 0 \\
0 & F(x)-\frac{1}{2} F(Q)_{n-1, n-1}
\end{array}\right]+\gamma_{n}\left[\begin{array}{cc}
-\left(\nabla_{Q} F\right)_{n, n-1} & \left(\nabla_{Q} F\right)_{n, n^{\star}} \\
-\left(\nabla_{Q} F\right)_{(n-1)^{\star}, n-1} & \left(\nabla_{Q} F\right)_{n, n-1}
\end{array}\right] \\
& \mathcal{U}^{(F), R}=\left[\begin{array}{cc}
0 & \mathcal{W}_{F} \\
0 & -F(x)
\end{array}\right]
\end{aligned}
$$

We now consider a deformation supported at one point $z=a$.

$$
\delta_{F}\left[p_{n}(x), \widetilde{p}_{n}(x)\right]=\mathbf{e}_{n}^{t} \cdot \mathbb{U}^{\left(\delta_{a}^{k}\right)} \cdot[\mathbf{p}, \widetilde{\mathbf{p}}]-\left.\mathbf{e}_{n}^{t}\left(\frac{\mathrm{d}}{\mathrm{d} z}\right)^{k}\right|_{z=a} \frac{\mathbf{p}(x)-\mathbf{p}(z)}{x-z}[0,1] .
$$

This time we have

$$
\begin{aligned}
& \mathbf{e}_{n}^{t} \cdot \mathbb{U}^{\left(\delta_{a}^{k}\right)} \cdot[\mathbf{p}, \widetilde{\mathbf{p}}]=\frac{1}{2} \partial_{a}^{k}\left(p_{n}(a) r_{n}(a)\right)\left[p_{n}, \widetilde{p}_{n}\right]+\partial_{a}^{k} \mathbf{e}_{n}^{t} \cdot \mathbf{p}(a) \mathbf{r}^{t}(a) \Pi_{n-1}[\mathbf{p}, \widetilde{\mathbf{p}}]= \\
= & \frac{1}{2} \partial_{a}^{k}\left(p_{n}(a) r_{n}(a)\right)\left[p_{n}, \widetilde{p}_{n}\right]+\partial_{a}^{k} \mathbf{e}_{n}^{t} \cdot \mathbf{p}(a)\left(\frac{\gamma_{n}\left[p_{n}^{\star}(a), r_{n-1}(a)\right]}{x-a} \mathbf{j} \chi_{n}(x)-\frac{[0,1]}{x-a}\right)
\end{aligned}
$$

We thus have

$$
=\frac{1}{2} \partial_{a}^{k}\left(p_{n}(a) r_{n}(a)\right)\left[p_{n}, \widetilde{p}_{n}\right]+\partial_{a}^{k} \frac{\gamma_{n}\left[p_{n}(a) p_{n}^{\star}(a), p_{n}(a) r_{n-1}(a)\right]}{x-a} \mathbf{j} \chi_{n}(x)-\partial_{a}^{k} \frac{\left[0, p_{n}(x)\right]}{x-a}
$$


Similarly for the Laurent polynomials

$$
\delta_{F}\left[r_{n-1}(x), \widetilde{r}_{n-1}(x)\right]=\mathbf{e}_{n-1}^{t} \cdot \mathbb{U}^{\left(\delta_{a}^{k}\right) t} \cdot[\mathbf{r}, \widetilde{\mathbf{r}}]-\left.\mathbf{e}_{n-1}^{t}\left(\frac{\mathrm{d}}{\mathrm{d} z}\right)^{k}\right|_{z=a} \frac{\mathbf{r}(x)-\mathbf{r}(z)}{x-z}[0,1],
$$

where now

$$
\begin{array}{r}
\mathbf{e}_{n-1}^{t} \cdot \mathbb{U}^{\left(\delta_{a}^{k}\right) t} \cdot[\mathbf{r}, \widetilde{\mathbf{r}}]= \\
=-\frac{1}{2} \partial_{a}^{k}\left(p_{n-1}(a) r_{n-1}(a)\right)\left[r_{n-1}, \widetilde{r}_{n-1}\right]+\partial_{a}^{k} \mathbf{e}_{n-1}^{t} \cdot \mathbf{r}(a)\left(\frac{\gamma_{n}\left[p_{n}(a), r_{n-1}^{\star}(a)\right]}{x-a} \mathbf{j} \chi_{n}^{\star t}(x)-\frac{[0,1]}{x-a}\right)
\end{array}
$$

so that finally

$$
\begin{aligned}
\delta_{F}\left[r_{n-1}(x), \widetilde{r}_{n-1}(x)\right]= & -\frac{1}{2} \partial_{a}^{k}\left(p_{n-1}(a) r_{n-1}(a)\right)\left[r_{n-1}, \widetilde{r}_{n-1}\right]+ \\
& +\partial_{a}^{k} \frac{\gamma_{n}\left[r_{n-1}(a) p_{n}(a), r_{n-1}(a) r_{n-1}^{\star}(a)\right]}{x-a} \mathbf{j} \chi_{n}^{\star}(x)-\partial_{a}^{k} \frac{\left[0, r_{n-1}(x)\right]}{x-a}
\end{aligned}
$$

Starifying this last identity and collecting it together with (6-21) we finally have

$$
\begin{aligned}
& \delta \chi_{n}(a)=\mathcal{U}_{n}^{\left(\delta_{a}^{k}\right)}(x) \chi_{n}(x)+\chi_{n}(x) \mathcal{U}^{\left(\delta_{a}^{k}\right), R}(x) \\
& \mathcal{U}_{n}^{\left(\delta_{a}^{k}\right)}(x)=\frac{\partial^{k}}{\partial a^{k}}\left\{\frac{1}{2}\left[\begin{array}{cc}
p_{n}(a) r_{n}(a) & 0 \\
0 & -p_{n-1}(a) r_{n-1}(a)
\end{array}\right]+\frac{\gamma_{n}}{x-a}\left[\begin{array}{cc}
p_{n}(a) p_{n}^{\star}(a) & p_{n}(a) r_{n-1}(a) \\
r_{n-1}(a) p_{n}(a) & r_{n-1}(a) r_{n-1}^{\star}(a)
\end{array}\right] \mathbf{j}\right\} \\
& =\frac{\partial^{k}}{\partial a^{k}} \frac{1}{2}\left[\begin{array}{cc}
p_{n} r_{n} & 0 \\
0 & -p_{n-1} r_{n-1}
\end{array}\right]_{z=a}+\frac{\partial^{k}}{\partial a^{k}} \frac{\gamma_{n}}{x-a}\left[\begin{array}{cc}
-p_{n} r_{n-1} & p_{n} p_{n}^{\star} \\
-r_{n-1} r_{n-1}^{\star} & r_{n-1} p_{n}
\end{array}\right]_{z=a} \\
& \mathcal{U}^{\left(\delta_{a}^{k}\right), R}(x)=\partial_{a}^{k}\left[\begin{array}{cc}
0 & \frac{1}{a-x} \\
0 & 0
\end{array}\right]
\end{aligned}
$$

This concludes the proof. Q.E.D.

\section{Moment functionals of integral type and ODE}

We now assume that the moment functional that we are considering admits an actual integral representation

$$
\mathcal{L}\left(z^{k}\right):=\sum \varkappa_{j} \int_{\Gamma_{j}} \mathrm{e}^{-V(z)} z^{k} \mathrm{~d} z
$$

As far as the previous discussion on deformations is concerned, the integral representation of the moment functional is largely irrelevant, the only issue being the convergence of the deformation function: therefore the "potential" $V(z)$ as well as the sets of integration $\Gamma_{j}$ could be completely arbitrary. However, in view of our intentions, we will assume that $\Gamma_{j}$ are contours in the complex plane and that $V(z)$ is a locally defined smooth function on these contours with the only restriction coming from the fact that negative moments should be defined as well as the positive ones.

In fact -although many considerations would remain identical in more general situations- we will assume that $V$ is a locally analytic function in the complex $z$-plane excepted at some punctures, identically to the case of semiclassical moment functionals studied in [5, 4] with the only extra restriction that all negative moments should be defined and finite. 
Semiclassical Moment Functionals. For the reader's convenience we briefly recall how these semiclassical moment functionals are constructed [5, 4, 17, 18. In this case the potential is such that the derivative is an arbitrary rational function

$$
V^{\prime}(z)=\text { Rational function }
$$

and thus $V(z)$ is a rational function plus logarithmic singularities at those poles of $V^{\prime}$ where the residue does not vanish. For simplicity we assume that $V^{\prime}$ has either a pole or a nonzero limit at $z=\infty$. Once we have chosen the potential $V$ we also choose an arbitrary collection of contours (avoiding $z=0)\left\{\Gamma_{j}\right\}$ with the property that $\Re(V(x))$ is uniformly bounded from below on all the chosen contours and tends to $\infty$ polynomially (in the length parameter) on the contours that extend to $z=\infty$. In more detailed terms:

(a) Consider a pole $z=c$ of $V^{\prime}$ of order $k \geq 2$ : we attach to it $k-1$ "petals" approaching $z=c$ along asymptotic directions in the sectors where $\Re(V(x)) \rightarrow+\infty$. We also attach a "stem" extending to $\infty$ and asymptotic to a direction such that $\Re(V(x)) \rightarrow \infty$.

(b) For a simple pole $z=c$ of $V^{\prime}$, if the residue is a positive integer (i.e. $\mathrm{e}^{-V}$ has a pole at $z=c$ ) we choose a small loop around the point, if the residue is a negative integer we take a contour from $z=c$ to $\infty$, if the residue is non integer we take a loop coming from $\infty$ and returning to $\infty$ (with the same restriction as above for the asymptotic direction).

(c) We choose also arbitrary segments joining a certain number of points $z=a$ to $\infty$ (along admissible directions). These latter contours are called "hard-edge" contours because the pseudo measure $\mathrm{d} \mu=\mathrm{e}^{-V(z)} \mathrm{d} z$ has a limit at $z=a$ and integration by parts yields a boundary term.

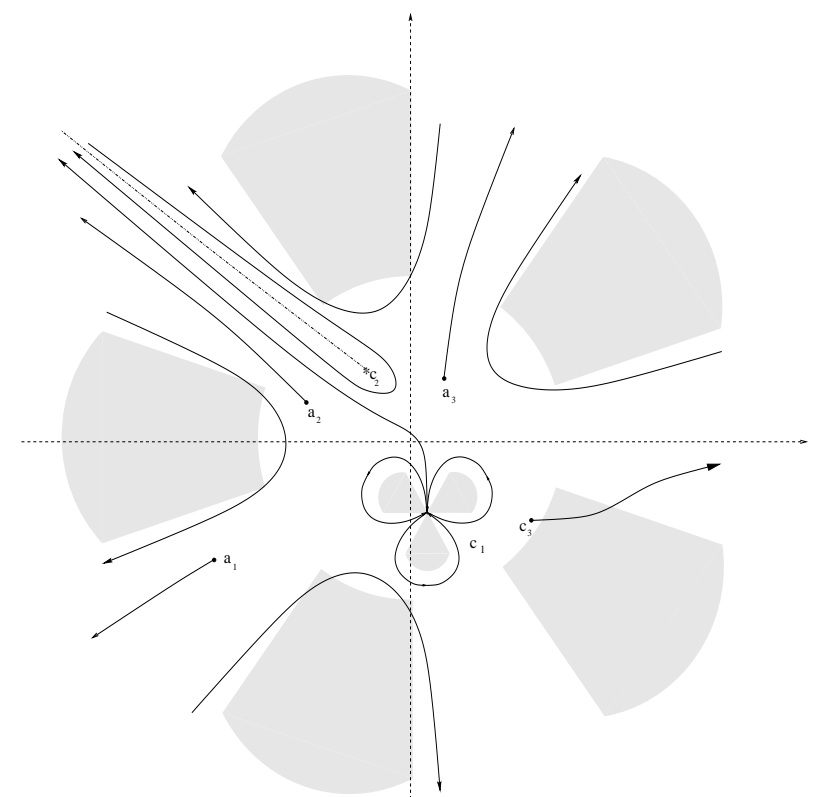

Figure: The contours for a typical semiclassical moment functional. Here $V^{\prime}(x)$ has a pole of order 4 at $\infty$, of order 4 at $c_{3}$ and simple poles at $c_{2}, c_{3}$ with noninteger and negative-integer residue respectively. The contours originating from the $a_{i}$ 's are "hard-edge" contours. The shaded sectors represent the asymptotic "forbidden" directions for approaching a singularity. One of these sectors at $\infty$ in Figure does not have a contour surrounding it because such a contour would be "homologically" equivalent to minus the sum of all others. 


\subsection{Differential equations}

We first analyze in this situation the infinite-dimensional differential equation that the BOPs satisfy. The natural differential operation in this setting is not $\partial_{x}$ but rather $x \partial_{x}$. Using the recurrence relations involving multiplication by $x$ and the orthogonality relations

$$
\begin{aligned}
& x \mathbf{p}(x)=Q \mathbf{p}(x) ; x \mathbf{r}^{t}(x)=\mathbf{r}^{t}(x) Q \\
& x \mathbf{p}^{\prime}(x)=D \mathbf{p}(x) ; x \mathbf{r}^{\prime}(x)=\tilde{D} \mathbf{r}(x) \\
& \int_{\varkappa} \mathbf{p r}^{t} \mathrm{e}^{-V} \mathrm{~d} z=\mathbf{1},
\end{aligned}
$$

we can obtain the following identity by integrating $\partial_{z}\left(z \mathbf{p r}^{t} \mathrm{e}^{-V(z)}\right)$ :

$$
D+\tilde{D}^{t}-\left.\left(z \mathbf{p}(z) \mathbf{r}^{t}(z) \mathrm{e}^{-V(z)}\right)\right|_{\partial \varkappa}=Q V^{\prime}(Q)-\mathbf{1}
$$

The notation that we now adopt is that $\int_{\varkappa}$ stands for the linear combination with coefficients $\varkappa_{j}$ of integrals on the oriented contours $\Gamma_{j}$ and the evaluations $\left.\right|_{\partial \varkappa}$ stand for the evaluations at all endpoints of the given contours, multiplied by the corresponding coefficient $\varkappa$ and the appropriate sign according to the orientation. The matrices $D$ and $\tilde{D}$ are lower triangular and on the main diagonal they can be explicitly computed

$$
\begin{aligned}
& x p_{n}^{\prime}=n p_{n}+\text { previous } \\
& x r_{n}^{\prime}=\left((-n)\left(1-\dot{\ell}_{n+1}\right)+\ell_{n+1}\right) r_{n}+\text { previous } \\
& x \frac{\mathrm{d}}{\mathrm{d} x} r_{n}^{\star}=n \dot{\ell}_{n+1} r_{n}^{\star}+\text { previous } .
\end{aligned}
$$

This implies the following Virasoro scaling constraint

$$
\left(Q V^{\prime}(Q)\right)_{n n}+\left.\left(z p_{n} r_{n} \mathrm{e}^{-V}\right)\right|_{\partial \varkappa}=1+\ell_{n+1}+n \dot{\ell}_{n+1} .
$$

Note that we also have

$$
\sum_{k=0}^{n-1}\left(\left(Q V^{\prime}(Q)\right)_{k k}+\left.\left(z p_{k} r_{k} \mathrm{e}^{-V}\right)\right|_{\partial \varkappa}\right)=\sum_{k=0}^{n-1}\left(1+\ell_{l+1}+l \dot{\ell}_{k+1}\right)=n\left(\ell_{n}+1\right) .
$$

The parts of $D, \widetilde{D}$ below the main diagonal are now expressed in terms of $Q$ and the boundary terms only

$$
\begin{aligned}
& D_{<}=\left(Q V^{\prime}(Q)\right)_{<}+\left.\left(z\left(\mathbf{p}(z) \mathbf{r}^{t}(z)\right)_{<} \mathrm{e}^{-V(z)}\right)\right|_{\partial \varkappa} \\
& \widetilde{D}_{<}=\left(Q^{t} V^{\prime}\left(Q^{t}\right)\right)_{<}+\left.\left(z\left(\mathbf{r}(z) \mathbf{p}^{t}(z)\right)_{<} \mathrm{e}^{-V(z)}\right)\right|_{\partial \varkappa}
\end{aligned}
$$

Note that -below the main diagonal- the matrices $D$ and $\widetilde{D}$ are of the same form as the deformations we were considering previously; more precisely they correspond to a variation by $F(z)=z V^{\prime}(z)$ and a linear combination of variations supported at the endpoints of the contours $\Gamma_{j}$. The folded version of this ODE can be obtained from the formulæ 6-9, 6-14, 6-21] 6-23) with the only modification that comes from the diagonal part of $D$. Using (7-10) for the diagonal part the reader can check that the result is

$$
\mathcal{D}_{n}=\left[\begin{array}{cc}
n & 0 \\
0 & x V^{\prime}(x)-1-\ell_{n}
\end{array}\right]+\gamma_{n}\left[\begin{array}{cc}
-W_{n, n-1} & W_{n, n^{\star}} \\
-W_{(n-1)^{\star}, n-1} & W_{n, n-1}
\end{array}\right]+\left.\left(\frac{z \mathrm{e}^{-V(z)} \gamma_{n}}{x-z}\left[\begin{array}{cc}
-p_{n} r_{n-1} & p_{n} p_{n}^{\star} \\
-r_{n-1} r_{n-1}^{\star} & r_{n-1} p_{n}
\end{array}\right]\right)\right|_{\partial \varkappa}
$$




$$
W:=\nabla_{Q} x V^{\prime}(x)=\frac{Q V^{\prime}(Q)-x V^{\prime}(x)}{Q-x}
$$

We remark that the last "boundary" term consists of simple poles with nilpotent residues.

For the full matrix $\chi_{n}$ the differential equation is

$$
\begin{aligned}
x \partial_{x} \chi_{n}(x) & =\mathcal{D}_{n}(x) \chi_{n}(x)+\chi_{n}(x) \mathcal{D}^{R}(x) \\
\mathcal{D}^{R}(x) & =\left[\begin{array}{lll}
0 & \int_{\varkappa} \frac{x V^{\prime}(x)-z V^{\prime}(z)}{x-z} \mathrm{e}^{-V(z)} \mathrm{d} z+\left.\frac{z \mathrm{e}^{-V(z)}}{z-x}\right|_{\partial \varkappa} \\
0 & -x V^{\prime}(x)
\end{array}\right]
\end{aligned}
$$

Together with the differential equation and the deformation equations we recall that we also have difference equations

$$
\begin{aligned}
\chi_{n} & =R_{n}(x) \chi_{n-1}, \quad n \geq 1 \\
R_{n}(x) & = \begin{cases}{\left[\begin{array}{cc}
\frac{x-\beta_{n}}{\gamma_{n}} & \kappa_{n} \\
(-1)^{n+1} & 0
\end{array}\right] \quad \text { if } \dot{\ell}_{n}=1} \\
{\left[\begin{array}{cc}
\frac{x-\beta_{n}}{\gamma_{n}} & \kappa_{n} \\
(-1)^{n+1} & \omega_{n}
\end{array}\right]} & \text { if } \dot{\ell}_{n}=0\end{cases}
\end{aligned}
$$

The ladder matrices $R_{n}$ are simply obtained from the transfer matrices (3-2) by using the normalization of the polynomials as in (4-5). We have thus proved

Theorem 7.1 The matrix $\chi_{n}$ satisfies the following system of difference-deformation-differential (DDD for short) equations

$$
\begin{aligned}
\chi_{n} & =R_{n}(x) \chi_{n-1} \\
x \frac{\mathrm{d}}{\mathrm{d} x} \chi_{n} & =\mathcal{D}_{n} \chi_{n}+\chi_{n} \mathcal{D}^{R} \\
\delta_{f} \chi_{n} & =\mathcal{U}_{n}^{(f)} \chi_{n}+\chi_{n} \mathcal{U}^{(f), R}
\end{aligned}
$$

where $f$ denotes either any function or formal power series provided that $\mathcal{L}\left(f(z) z^{k}\right)$ is well defined for $k \in \mathbb{Z}$ or any derivative of the Dirac delta function supported at any point $a \neq 0$.

We observe that the right action of the differential-deformation equation is independent of $n$. This suggests that we can perform a "right gauge" change to dispose of this part. Indeed we define the new object $\boldsymbol{\Gamma}_{n}$ which will be the focus in the rest of the paper

$$
\boldsymbol{\Gamma}_{n}:=\chi_{n}\left[\begin{array}{cc}
1 & -\mathrm{e}^{V(x)} \int_{\varkappa} \frac{\mathrm{e}^{-V(z)}}{x-z} \mathrm{~d} z \\
0 & \mathrm{e}^{V(x)}
\end{array}\right]
$$

It is easy to verify that this change of gauge eliminates the right-actions for the differential equation and for any deformation of $V(x)$ and/or the endpoints of integration. The first column of $\boldsymbol{\Gamma}_{n}$ is the same as the first column of $\chi_{n}$ and hence contains the LOPs. The second column contains now the following auxiliary functions

$$
\begin{aligned}
\psi_{n} & =\mathrm{e}^{V(x)} \int_{\varkappa} \frac{p_{n}(z) \mathrm{e}^{-V(z)}}{x-z} \mathrm{~d} z \\
\phi_{n-1}^{\star} & =x^{n-1-\ell_{n}} \phi_{n-1}=x^{n-1-\ell_{n}} \mathrm{e}^{V(x)} \int_{\varkappa} \frac{r_{n-1}(z) \mathrm{e}^{-V(z)}}{x-z} \mathrm{~d} z .
\end{aligned}
$$


We note that the auxiliary functions are piecewise analytic functions off the contours $\Gamma_{j}$ : it is a matter of routine inspection to read-off the relevant Riemann-Hilbert data. We defer this inspection to a later section.

In terms of the matrices $\boldsymbol{\Gamma}_{n}$ we have a DDD system of more standard form, without right multipliers.

Theorem 7.2 The following system of Difference-Differential-Deformation equations is Frobenius compatible

$$
\begin{aligned}
\boldsymbol{\Gamma}_{n} & =R_{n}(x) \boldsymbol{\Gamma}_{n-1} \\
x \frac{\mathrm{d}}{\mathrm{d} x} \boldsymbol{\Gamma}_{n} & =\mathcal{D}_{n} \boldsymbol{\Gamma}_{n} \\
\delta_{f} \boldsymbol{\Gamma}_{n} & =\mathcal{U}_{n}^{(f)} \boldsymbol{\Gamma}_{n}
\end{aligned}
$$

where $f$ is as in Thm. 7.1.).

A few remark are in order here: by choosing $f$ in Thm. (7.2) to be an ordinary function one can vary the potential $V$ by $V \rightarrow V+\epsilon f$ and hence all flows of the generalized Toda hierarchy are here included. However we can also choose $f$ as a distribution $\delta_{a}^{(k)}$ or linear combinations thereof. Clearly if we choose the point $a$ arbitrarily outside of the singularities of $V(x)$ we still have a compatibility of the resulting system but we will change the structure of the singularities of $\mathcal{D}_{n}$, which falls outside of the standard theory of isomonodromic deformations. For example, adding a $\delta_{a}$ corresponds to adding a term $\ln (x-a)$ in the potential and adjoining a small circle around $a$ to the set of contours $\Gamma_{j}$ 's.

Vice-versa the cases in which $f$ is a distribution which does not alter the singularity structure of $\mathcal{D}_{n}$ are:

1. Movement of the endpoints which contribute to the boundary term ${ }^{6}$ : then we have

$$
f= \pm \varkappa \mathrm{e}^{-V(a)} \delta_{a}
$$

where the coefficient $\varkappa$ is the coefficient of the contour $\Gamma_{j}$ which has $a$ as endpoint and the sign depends on the orientation of $\Gamma_{j}$.

2. Movements of poles of order $k$ (if any) of the pseudo-measure $\mathrm{e}^{-V} \mathrm{~d} z$ : then we have

$$
f(a)= \pm \varkappa k \delta_{a}^{(k+1)}(z) \mathrm{e}^{-V_{r}(z)}
$$

where the coefficient $\varkappa$ is the coefficient of the loop encircling $a$, the sign is chosen according to the orientation of the contour and $V_{r}(z)$ is the part of $V$ which is regular at $z=a$.

\section{Spectral curve and Isomonodromic tau function}

The objective of this section is that of expressing the spectral curve of the connection $\partial_{x}-\frac{1}{x} \mathcal{D}_{n}(x)$ in terms of the logarithmic derivatives of the Töplitz determinants; this will be the essential bridge to connect with the isomonodromic tau function in the coming sections. We prove the following theorem

Theorem 8.1 The following formula holds

$$
\begin{aligned}
\operatorname{det}\left(y \mathbf{1}-\frac{1}{x} \mathcal{D}_{n}(x)\right)= & y^{2}-\left(V^{\prime}(x)+\frac{L_{n}}{x}\right) y+ \\
& +\frac{1}{x} \operatorname{Tr}_{n}\left(\frac{Q V^{\prime}(Q)-x V^{\prime}(x)}{Q-x}\right)+\left.\frac{1}{x}\left(\frac{z \mathrm{e}^{-V(z)} \mathbf{p}^{t} \Pi_{n-1} \mathbf{r}}{x-z}\right)\right|_{\partial \varkappa} \\
& L_{n}:=n-1-\ell_{n},
\end{aligned}
$$

\footnotetext{
${ }^{6}$ They corresponds to those endpoints of the contours $\Gamma_{j}$ for which $\lim _{\Gamma_{j} \ni z \rightarrow \partial \Gamma_{j}} \mathrm{e}^{-V(z)} \neq 0$.
} 
where $\Pi_{n-1}=\operatorname{diag}(1,1, \ldots, 1,0, \ldots)$ ( $n$ nonzero entries).

Before proceeding to the proof we remark that this formula would be valid for an arbitrary smooth potential; quite clearly, however, in this case the spectral curve would not be an algebraic curve.

Proof.

We need to compute the two spectral invariants of the connection; the main tool is to use the compatibility between the ladder relations and the connections $\mathcal{D}_{n}(x)$. Indeed from the compatibility between the difference and differential equation and from the explicit expression for $\mathcal{D}_{n}(x)$ (7-14) we can express recurrence relation for the spectral invariants of $\mathcal{D}_{n}(x)$. The trace is computed by sight

$$
\operatorname{Tr}\left(\mathcal{D}_{n}(x)\right)=x V^{\prime}(x)+n-1-\ell_{n}
$$

From the compatibility of difference-differential equations we have the gauge property

$$
\mathcal{D}_{n-1}=R_{n}{ }^{-1} \mathcal{D}_{n} R_{n}-x R_{n}{ }^{-1} R_{n}^{\prime}
$$

The gauge term is explicitly computed to be

$$
\begin{aligned}
& R_{n}{ }^{-1} \mathcal{D}_{n} R_{n}=\mathcal{D}_{n-1}+x R_{n}{ }^{-1} R_{n}^{\prime} \\
& x R_{n}{ }^{-1} R_{n}^{\prime}= \begin{cases}(-1)^{n} \frac{x}{\gamma_{n-1}}\left[\begin{array}{ll}
0 & 0 \\
1 & 0
\end{array}\right] & \text { if } \dot{\ell}_{n}=1 \\
{\left[\begin{array}{cc}
1 & 1 \\
\frac{(-1)^{n} \Delta_{n-1}^{\ell_{n}-1}}{\Delta_{n}^{\ell_{n}} \sqrt{\Delta_{n-2}^{\ell_{n-2}}}} & 0
\end{array}\right]} & \text { if } \dot{\ell}_{n}=0\end{cases}
\end{aligned}
$$

These formulæ imply a recurrence relation for the quadratic invariant.

$$
\left.\operatorname{Tr}\left(\mathcal{D}_{n}^{2}\right)\right)=\operatorname{Tr}\left(\mathcal{D}_{n-1}^{2}\right)+2 \operatorname{Tr}\left(\mathcal{D}_{n-1} x R_{n}{ }^{-1} R_{n}^{\prime}\right)+\operatorname{Tr}\left(\left(x R_{n}{ }^{-1} R_{n}^{\prime}\right)^{2}\right) .
$$

For the line case i.e. $\dot{\ell}_{n}=1$ and using the form of the recursion matrices $R_{n}$ together with the fact that in this case $r_{n-1}^{\star}=(-1)^{n-1} p_{n-1}$ we find

$$
\operatorname{Tr}\left(\mathcal{D}_{n}^{2}\right)=\operatorname{Tr}\left(\mathcal{D}_{n-1}^{2}\right)-2 x\left(\frac{Q V^{\prime}(Q)-x V^{\prime}(x)}{Q-x}\right)_{n-1, n-1}-\left.2 x\left(\frac{z \mathrm{e}^{-V(z) p_{n-1} r_{n-1}}}{x-z}\right)\right|_{\partial \varkappa}
$$

For the circle case $\dot{\ell}_{n}=0$ instead we have

$$
\begin{aligned}
\operatorname{Tr}\left(\mathcal{D}_{n}^{2}\right)= & \operatorname{Tr}\left(\mathcal{D}_{n-1}^{2}\right)+2(n-1)+2 \gamma_{n-1}\left(-W_{n-1, n-2}+\frac{(-1)^{n} \Delta_{n-1}^{\ell_{n}-1}}{\Delta_{n}^{\ell_{n}} \sqrt{\Delta_{n-2}^{\ell_{n-2}}}} W_{n-1,(n-1)^{\star}}\right)+ \\
& +\left.2 \gamma_{n-1}\left(\frac{z \mathrm{e}^{-V(z)} p_{n-1}}{x-z}\left(-r_{n-2}+\frac{(-1)^{n} \Delta_{n-1}^{\ell_{n}-1}}{\Delta_{n}^{\ell_{n}} \sqrt{\Delta_{n-2}^{\ell_{n-2}}}} p_{n-1}^{\star}\right)\right)\right|_{\partial \varkappa}+1
\end{aligned}
$$

Using the identity (2-7) together with the definitions of the biorthogonal polynomials and the various normalization factors (4-5) one can see that

$$
-r_{n-2}+\frac{(-1)^{n} \Delta_{n-1}^{\ell_{n}-1}}{\Delta_{n}^{\ell_{n}} \sqrt{\Delta_{n-2}^{\ell_{n-2}}}} p_{n-1}^{\star}=-\frac{z}{\gamma_{n-1}} r_{n-1},
$$


and hence

$$
-W_{n-1, n-2}+\frac{(-1)^{n} \Delta_{n-1}^{\ell_{n}-1}}{\Delta_{n}^{\ell_{n}} \sqrt{\Delta_{n-2}^{\ell_{n-2}}}} W_{n-1,(n-1)^{\star}}=\frac{1}{\gamma_{n-1}} \mathcal{L}_{z}\left(z W p_{n-1} r_{n-1}\right)
$$

Therefore the recursion for the circle case is

$$
\begin{aligned}
\operatorname{Tr}\left(\mathcal{D}_{n}^{2}\right)-\operatorname{Tr}\left(\mathcal{D}_{n-1}^{2}\right)= & 2(n-1)+1-\left.2\left(\frac{Q\left(Q V^{\prime}(Q)-x V^{\prime}(x)\right)}{Q-x}\right) \underset{n-1, n-1}{-2}\left(\frac{z^{2} \mathrm{e}^{-V(z)} p_{n-1} r_{n-1}}{x-z}\right)\right|_{\partial \varkappa}= \\
= & 2(n-1)+1-2\left(Q V^{\prime}(Q)_{n-1, n-1}+\left.\left(z \mathrm{e}^{-V(z)} p_{n-1} r_{n-1}\right)\right|_{\partial \varkappa}\right)+2 x V^{\prime}(x)+ \\
& -2 x\left(\frac{Q V^{\prime}(Q)-x V^{\prime}(x)}{Q-x}\right)_{n-1, n-1}-\left.2 x\left(\frac{z \mathrm{e}^{-V(z)} p_{n-1} r_{n-1}}{x-z}\right)\right|_{\partial \varkappa}= \\
= & 2(n-1)-1-2 \ell_{n}+2 x V^{\prime}(x)-2 x\left(\frac{Q V^{\prime}(Q)-x V^{\prime}(x)}{Q-x}\right)_{n-1, n-1}^{+}+ \\
& -\left.2 x\left(\frac{z \mathrm{e}^{-V(z)} p_{n-1} r_{n-1}}{x-z}\right)\right|_{\partial \varkappa}
\end{aligned}
$$

Summarizing, in the two cases we have found

$$
\begin{aligned}
\operatorname{Tr}\left(\mathcal{D}_{n}^{2}\right)-\operatorname{Tr}\left(\mathcal{D}_{n-1}^{2}\right)= & 2\left(x V^{\prime}(x)-\ell_{n}+(n-1)-\frac{1}{2}\right)\left(1-\dot{\ell}_{n}\right)+ \\
& -\left.2 x\left(\frac{Q V^{\prime}(Q)-x V^{\prime}(x)}{Q-x}\right)_{n-1, n-1}^{-2 x}\left(\frac{z \mathrm{e}^{-V(z)} p_{n-1} r_{n-1}}{x-z}\right)\right|_{\partial \varkappa}
\end{aligned}
$$

To complete the computation we need to find $\operatorname{Tr}\left(\mathcal{D}_{1}{ }^{2}\right)$ or -equivalently- $\operatorname{det}\left(\mathcal{D}_{1}\right)$. We have

$$
\begin{aligned}
\operatorname{det}\left(\frac{1}{x} D_{1}\right) & =\operatorname{det}\left[\begin{array}{cc}
p_{1}^{\prime} & \psi_{1}^{\prime} \\
r_{0}^{\star \prime} & \phi_{0}^{\star^{\prime}}
\end{array}\right]\left[\begin{array}{cc}
p_{1} & \psi_{1} \\
r_{0}^{\star} & \phi_{0}^{\star}
\end{array}\right]=\operatorname{det}\left[\begin{array}{cc}
p_{1}^{\prime} & \psi_{1}^{\prime} \\
r_{0}^{\star \prime} & \phi_{0}^{\star^{\prime}}
\end{array}\right] \mathrm{e}^{-V(x)}= \\
& =\sqrt{h_{1}} \mathrm{e}^{-V(x)} \operatorname{det}\left[\begin{array}{cc}
\frac{1}{\sqrt{h_{1}}} & \psi_{1}^{\prime} \\
0 & \phi_{0}^{\star \prime}
\end{array}\right]= \\
& =V^{\prime}(x) \mathcal{L}_{z}\left(\frac{1}{x-z}\right)-\mathcal{L}_{z}\left(\frac{V^{\prime}(z)}{x-z}\right)+\left.\left(\frac{\mathrm{e}^{-V(z)}}{x-z}\right)\right|_{\partial \varkappa}= \\
& =\left(\frac{V^{\prime}(Q)-V^{\prime}(x)}{Q-x}\right)_{00}+\left.\left(\frac{\mathrm{e}^{-V(z)} p_{0} r_{0}}{x-z}\right)\right|_{\partial \varkappa}
\end{aligned}
$$

This implies

$$
\begin{aligned}
\operatorname{det} \mathcal{D}_{1}(x) & =x^{2}\left(\frac{V^{\prime}(Q)-V^{\prime}(x)}{Q-x}\right)_{00}+\left.x^{2}\left(\frac{\mathrm{e}^{-V(z)} p_{0} r_{0}}{x-z}\right)\right|_{\partial \varkappa}= \\
& =x \overbrace{\left(V^{\prime}(Q)_{00}+\left.\left(p_{0} r_{0} \mathrm{e}^{-V(z)}\right)\right|_{\partial \varkappa}\right)}^{=0}+x\left(\frac{Q V^{\prime}(Q)-x V^{\prime}(x)}{Q-x}\right)_{00}+\left.x\left(\frac{z \mathrm{e}^{-V(z)} p_{0} r_{0}}{x-z}\right)\right|_{\partial \varkappa}= \\
& =x\left(\frac{Q V^{\prime}(Q)-x V^{\prime}(x)}{Q-x}\right)_{00}+\left.x\left(\frac{z \mathrm{e}^{-V(z)} p_{0} r_{0}}{x-z}\right)\right|_{\partial \varkappa}
\end{aligned}
$$


Hence $\left(\ell_{1}=0\right)$

$$
\begin{aligned}
\operatorname{Tr}\left(\mathcal{D}_{1}{ }^{2}\right)= & \left(x V^{\prime}(x)\right)^{2}-2 x\left(\frac{Q V^{\prime}(Q)-x V^{\prime}(x)}{Q-x}\right)_{00}-\left.2 x\left(\frac{z \mathrm{e}^{-V(z)} p_{0} r_{0}}{x-z}\right)\right|_{\partial \varkappa} \\
\operatorname{Tr}\left(\mathcal{D}_{n}{ }^{2}\right)= & \left(x V^{\prime}(x)\right)^{2}+2 x V^{\prime}(x)\left(n-1-\ell_{n}\right)-\left(n-1-\ell_{n}\right)+2 \sum_{k=1}^{n}\left(k-1-\ell_{k}\right)\left(1-\dot{\ell}_{k}\right)+ \\
& -2 x \operatorname{Tr}_{n}\left(\frac{Q V^{\prime}(Q)-x V^{\prime}(x)}{Q-x}\right)-\left.2 x\left(\frac{z \mathrm{e}^{-V(z)} \mathbf{p}^{t} \Pi_{N-1} \mathbf{r}}{x-z}\right)\right|_{\partial \varkappa}
\end{aligned}
$$

Using this expression for the quadratic invariant we can obtain the following formula for the characteristic polynomial

$$
\begin{aligned}
\operatorname{det}\left(\tilde{y} \mathbf{1}-\mathcal{D}_{n}(x)\right)= & \tilde{y}^{2}-\left(x V^{\prime}(x)+n-1-\ell_{n}\right) \tilde{y}+K_{n}+ \\
+ & +x \operatorname{Tr}_{n}\left(\frac{Q V^{\prime}(Q)-x V^{\prime}(x)}{Q-x}\right)+\left.x\left(\frac{z \mathrm{e}^{-V(z)} \mathbf{p}^{t} \Pi_{N-1} \mathbf{r}}{x-z}\right)\right|_{\partial \varkappa} \\
& K_{n}:=\frac{\left(n-1-\ell_{n}\right)\left(n-\ell_{n}\right)}{2}+\sum_{k=2}^{n}\left(\ell_{k}+1-k\right)\left(1-\dot{\ell}_{k}\right)
\end{aligned}
$$

The last crucial observation is that $K_{n} \equiv 0$ for all $n$ : this is non-obvious at first sight and it is true only because $\ell_{n}$ is a weakly increasing sequence of integers. Indeed one can check that

$$
K_{n+1}-K_{n}=\frac{1}{2} \dot{\ell}_{n+1}\left(1-\dot{\ell}_{n+1}\right),
$$

so that $K_{n+1}=K_{n}=K_{1}=0$. To conclude the proof we note that the spectral curve of (8-18) is simply related to that of the connection by $\tilde{y}=x y$. This ends our proof.

\section{Isomonodromic deformations}

By Thm. 7.2 we have compatible systems of Difference, Differential, Deformation equations

$$
\begin{aligned}
\boldsymbol{\Gamma}_{n} & =R_{n} \boldsymbol{\Gamma}_{n-1} \\
\partial_{x} \boldsymbol{\Gamma}_{n} & =\frac{1}{x} \mathcal{D}_{n} \boldsymbol{\Gamma}_{n} \\
\delta_{f} \boldsymbol{\Gamma}_{n} & =\mathcal{U}_{n}^{(f)}(x) \boldsymbol{\Gamma}_{n}
\end{aligned}
$$

The compatibility of this system entails isomonodromic deformations for the connection $\partial_{x}-\frac{1}{x} \mathcal{D}_{n}$. Note that this connection has the same singularity structure of $V^{\prime}(x)$. In order to have isomonodromic deformations in the sense of Miwa-Jimbo-Ueno we need to impose that $V^{\prime}(x)$ be a rational function. Then the deformations of $V(x)$ which give rise to the setting in MJU are those which do not alter the singularity structure of $V(x)$; this is why the most general setting compatible with this requirement is that of semiclassical moment functionals.

\subsection{Spectral residue-formulæ}

Mimicking the approach of 4 we can express the logarithmic derivatives of the shifted Töplitz determinants $\Delta_{n}^{\ell_{n}}$ in terms of residue formulæ involving the differential $y \mathrm{~d} x$ on the spectral curve defined in 
Thm. 7.2

$$
\begin{aligned}
Y_{ \pm}(x) & :=\frac{1}{2}\left(V^{\prime}(x)+\frac{L_{n}}{x}\right) \pm \frac{1}{2} \sqrt{\left(V^{\prime}(x)+\frac{L_{n}}{x}\right)^{2}-4 \mathcal{P}(x)} \\
\mathcal{P}(x) & =\frac{1}{x} \operatorname{Tr}_{n}\left(\frac{Q V^{\prime}(Q)-x V^{\prime}(x)}{Q-x}\right)+\left.\frac{1}{x}\left(\frac{z \mathrm{e}^{-V(z)} \mathbf{p}^{t} \Pi_{N-1} \mathbf{r}}{x-z}\right)\right|_{\partial \varkappa}
\end{aligned}
$$

Indeed we have

Theorem 9.1 Let $V^{\prime}(x)$ be rational.

(i) Suppose that $x=c$ is a pole of order $d+1$

$$
\begin{array}{r}
V(x)=\sum_{J=1}^{d} \frac{t_{J}^{(c)}}{J(x-c)^{J}}-t_{0}^{(c)} \ln (x-c)+\mathcal{O}(1) \\
V^{\prime}(x)=-\sum_{J=0}^{d} \frac{t_{J}^{(c)}}{(x-c)^{J+1}}+\mathcal{O}(1)
\end{array}
$$

Then we have

$$
\begin{aligned}
t_{J}^{(c)} & =-\operatorname{res}_{x=c} Y_{+}(x)(x-c)^{J} \mathrm{~d} x, \quad J=0, \ldots d \\
\frac{\partial \ln \Delta_{n}^{\ell_{n}}}{\partial t_{J}^{(c)}} & =\frac{1}{J} \underset{x=c}{\operatorname{res}} Y_{-}(x)(x-c)^{-J} \mathrm{~d} x, \quad J=1, \ldots, d \\
\frac{\partial \ln \Delta_{n}^{\ell_{n}}}{\partial c} & =\operatorname{res}_{x=c} Y_{-}(x)\left(\sum_{J=0}^{d} \frac{t_{J}^{(c)}}{(x-c)^{J+1}}\right) \mathrm{d} x
\end{aligned}
$$

(ii) Suppose that $x=\infty$ is a pole of $V^{\prime}$ with degree d, namely

$$
\begin{array}{r}
V(x)=\sum_{J=1}^{d+1} \frac{t_{J}^{(\infty)}}{J} x^{J}+\mathcal{O}(\ln x) \\
V^{\prime}(x)=\sum_{J=1}^{d+1} t_{J}^{(\infty)} x^{J-1}+\mathcal{O}(1 / x)
\end{array}
$$

Then we have

$$
\begin{aligned}
& t_{J}^{(\infty)}=-\operatorname{res}_{x=\infty} Y_{+}(x) x^{-J} \mathrm{~d} x, \quad J=1, \ldots d+1 \\
& \frac{\partial \ln \Delta_{n}^{\ell_{n}}}{\partial t_{J}^{(\infty)}}=\frac{1}{J} \underset{x=\infty}{\operatorname{res}} x^{J} Y_{-}(x) \mathrm{d} x, \quad J=1, \ldots, d+1
\end{aligned}
$$

(iii) Let $x=a$ be $a$ hard-edge ${ }^{7}$, namely a point of the boundary of one of the contours $\left\{\Gamma_{j}\right\}$ such that $|V(a)|<\infty$. Then

$$
\frac{\partial \ln \Delta_{n}^{\ell_{n}}}{\partial a}=\frac{1}{2} \underset{x=a}{\operatorname{res}} \frac{1}{x^{2}} \operatorname{Tr}\left(\mathcal{D}_{n}\right)^{2} \mathrm{~d} x
$$

\footnotetext{
${ }^{7}$ This means that this is one of the points contributing to the boundary terms.
} 
(iv) Finally we have

$$
\begin{aligned}
& \underset{x=0}{\operatorname{res}} Y_{+}(x) \mathrm{d} x=L_{n}=n-1-\ell_{n}-\sum_{c=\text { finite pole of } V^{\prime}} t_{0}^{(c)} \\
& \underset{x=\infty}{\operatorname{res}} Y_{+}(x) \mathrm{d} x=\ell_{n}+1+t_{0}^{(\infty)}
\end{aligned}
$$

Proof. We start by noticing that

$$
Y_{ \pm}=\left\{\begin{array}{l}
1 \\
0
\end{array}\right\}\left(V^{\prime}(x)+\frac{L_{n}}{x}\right) \mp \frac{\mathcal{P}(x)}{V^{\prime}(x)+\frac{L_{n}}{x}}+\left\{\begin{array}{cc}
\mathcal{O}\left((x-c)^{d+1}\right) & \text { for case (i) } \\
\mp \frac{n^{2}}{t_{d+1}^{(\infty)} x^{d+1}}+\mathcal{O}\left(x^{-d-2}\right) & \text { for case (ii) }
\end{array}\right.
$$

At this point formulæ (9-8) 9-13 9-16) follow immediately by noticing that $\mathcal{P} /\left(V^{\prime}(x)+L_{n} / x\right)=\mathcal{O}(1)$ in all cases and by straightforward computation of residues ${ }^{8}$. As for the remaining formulæ we have, for case (i)

$$
\begin{gathered}
\underset{x=c}{\operatorname{res}}(x-c)^{-J} Y_{-}(x) \mathrm{d} x=\underset{x=c}{\operatorname{res}}(x-c)^{-J} \frac{\mathcal{P}(x)}{V^{\prime}(x)+\frac{L_{n}}{x}}=\underset{x=c}{\operatorname{res}} \frac{(x-c)^{-J}}{x V^{\prime}(x)+L_{n}} \operatorname{Tr}_{n}\left(\frac{x V^{\prime}(x)-Q V^{\prime}(Q)}{x-Q}\right)= \\
=\sum_{k=0}^{n-1} \mathcal{L}_{z}\left[\underset{x=c}{\operatorname{res}} \frac{(x-c)^{-J}}{x V^{\prime}(x)+L_{n}} \frac{x V^{\prime}(x)-z V^{\prime}(z)}{x-z} p_{n}(z) r_{n}(z)\right]= \\
=-\operatorname{Tr}_{n}(Q-c)^{-J}=J \partial_{t_{J}^{(c)}} \ln \Delta_{n}^{\ell_{n}}, \quad J=1, \ldots, d,
\end{gathered}
$$

and similar computation for the $c$-derivative. Here we have used the formulæ $5-12$ expressing the variation of $\ln \Delta_{n}^{\ell_{n}}$ under an infinitesimal deformation of the type ensuing from an infinitesimal change of the parameters $t_{J}^{(c)}$.

For case (ii) the computation is completely parallel except for the last $J=d+1$ residue. Indeed

$$
\begin{gathered}
\underset{x=\infty}{\operatorname{res}} x^{J} Y_{-}(x) \mathrm{d} x=\operatorname{res}_{x=\infty} x^{J} \frac{\mathcal{P}(x)}{V^{\prime}(x)+\frac{L_{n}}{x}}=\underset{x=\infty}{\operatorname{res}} \frac{x^{J}}{x V^{\prime}(x)+L_{n}} \operatorname{Tr}_{n}\left(\frac{x V^{\prime}(x)-Q V^{\prime}(Q)}{x-Q}\right)= \\
=\sum_{k=0}^{n-1} \mathcal{L}_{z}\left[\operatorname{rrs}_{x=\infty} \frac{x^{J}}{x V^{\prime}(x)+L_{n}} \frac{x V^{\prime}(x)-z V^{\prime}(z)}{x-z} p_{n}(z) r_{n}(z)\right]= \\
=-\operatorname{Tr}_{n} Q^{J}=J \partial_{t_{J}^{(\infty)}} \ln \Delta_{n}^{\ell_{n}}, \quad J=1, \ldots, d .
\end{gathered}
$$

For $J=d+1$ one has to use a similar manipulation but has to use the refined asymptotics (9-17): indeed we have

$$
\begin{array}{r}
\underset{x=\infty}{\operatorname{res}} x V^{\prime}(x) Y_{-}(x) \mathrm{d} x=-\operatorname{Tr}_{n} Q V^{\prime}(Q)+\left(n^{2}-n L_{n}-\operatorname{Tr}_{n} Q V^{\prime}(Q)-\left.z \mathrm{e}^{-V(z)} \mathbf{p}^{t} \Pi_{n-1} \mathbf{r}\right|_{\partial \varkappa}\right)= \\
=-\operatorname{Tr}_{n} Q V^{\prime}(Q)
\end{array}
$$

${ }^{8}$ Note that at infinity

$$
\frac{\mathcal{P}(x)}{V^{\prime}(x)+\frac{L_{n}}{x}}=\frac{n}{x}+\mathcal{O}\left(x^{-2}\right)
$$


where we have used (7-11) together with the definition of $L_{n}=n-1-\ell_{n}$. This proves, together with the residues (9-20)

$$
\underset{x=\infty}{\operatorname{res}} x^{d+1} Y_{-}(x) \mathrm{d} x=-\operatorname{Tr}_{n} Q^{d+1}=(d+1) \partial_{t_{d+1}^{(\infty)}} \ln \Delta_{n}^{\ell_{n}} .
$$

Finally, for the case (iii) the computation is immediate using the formula for $\operatorname{Tr} \mathcal{D}_{n}^{2}$ (8-17). Q. E. D.

\section{Riemann-Hilbert problem, Tau function}

Direct inspection of the asymptotic behavior of the biorthogonal polynomials and second kind functions allows us to ascertain the Riemann-Hilbert data for this problem. We start by noticing the following formal asymptotic behavior of the auxiliary functions entering in $\boldsymbol{\Gamma}_{n}$

$$
\begin{gathered}
\psi_{n}=\mathrm{e}^{V(x)} \int_{\varkappa} \frac{\mathrm{e}^{-V(z)} p_{n}(z)}{x-z}= \begin{cases}(-)^{n} x^{-\ell_{n}-2} \mathrm{e}^{V(x)} \sqrt{\frac{\Delta_{n+1}^{\ell_{n}+1}}{\Delta_{n}^{\ell_{n}}}}\left(1+\mathcal{O}\left(x^{-1}\right)\right) & \text { for } x \rightarrow \infty \\
-x^{n-1-\ell_{n}} \mathrm{e}^{V(x)} \frac{\Delta_{n+1}^{\ell_{n}}}{\sqrt{\Delta_{n}^{\ell_{n}} \Delta_{n+1}^{\ell_{n}}}}(1+\mathcal{O}(x)) & \text { for } x \rightarrow 0 \\
\mathrm{e}^{V(x)} \sqrt{h_{0}}(Q-c)_{n 0}^{-1} & \text { near poles of } V^{\prime}(x)\end{cases} \\
\phi_{n-1}^{\star}=x^{n-1-\ell_{n}} \mathrm{e}^{V(x)} \int_{\varkappa} \frac{\mathrm{e}^{-V(z)} r_{n-1}(z)}{x-z}= \begin{cases}x^{-\ell_{n}-1} \mathrm{e}^{V(x)} \sqrt{\frac{\Delta_{n}^{\ell_{n}}}{\Delta_{n-1}^{\ell_{n-1}}}\left(1+\mathcal{O}\left(x^{-1}\right)\right)} & \text { for } x \rightarrow \infty \\
x^{n-1-\ell_{n}} \mathrm{e}^{V(x)} \frac{(-)^{n} \Delta_{n}^{\ell_{n}-1}}{\sqrt{\Delta_{n}^{\ell_{n}} \Delta_{n-1}^{\ell_{n-1}}}}(1+\mathcal{O}(x)) & \text { for } x \rightarrow 0 \\
\mathrm{e}^{V(x)} \sqrt{h_{0}} c^{n-\ell_{n}+1}(Q-c)_{0, n-1}^{-1} & \text { near poles of } V^{\prime}(x)\end{cases}
\end{gathered}
$$

where we have used the definition of the LOPs (4-5) and the facts that

$$
\begin{array}{rll}
p_{n} \propto \wp_{n}^{\ell_{n}} & \perp z^{\ell_{n}-n+1}, \ldots, z^{\ell_{n}} \\
r_{n-1} \propto z^{\ell_{n}-n+1} \wp_{n-1}^{\ell_{n}-1} & \perp z^{0}, \ldots, z^{n-2}
\end{array}
$$

This implies the following formal asymptotic data for $\boldsymbol{\Gamma}_{n}$ near all the singularities.

At $x=0$ we have

$$
\begin{array}{r}
\boldsymbol{\Gamma}_{n}(x) \sim G_{n}^{(0)}\left[\begin{array}{ll}
1 & 0 \\
0 & x^{n-1-\ell_{n}} \mathrm{e}^{V_{\text {sing }, 0}(x)}
\end{array}\right](\mathbf{1}+\mathcal{O}(x)) \\
G_{n}^{(0)}:=\left[\begin{array}{cc}
\frac{(-)^{n} \Delta_{n}^{\ell_{n}+1}}{\sqrt{\Delta_{n}^{\ell_{n}} \Delta_{n+1}^{\ell_{n}+1}}} & -\frac{\Delta_{n+1}^{\ell_{n}}}{\sqrt{\Delta_{n}^{\ell_{n}} \Delta_{n+1}^{\ell_{n}+1}}} \\
\frac{\Delta_{n-1}^{\ell_{n}}}{\sqrt{\Delta_{n-1}^{\ell_{n}-1} \Delta_{n}^{\ell_{n}}}} & \frac{(-)^{n} \Delta_{n}^{\ell_{n}-1}}{\sqrt{\Delta_{n-1}^{\ell_{n}-1} \Delta_{n}^{\ell_{n}}}}
\end{array}\right], \operatorname{det} G_{n}^{(0)}=\frac{1}{\Delta_{n+1}^{\ell_{n+1}} \Delta_{n-1}^{\ell_{n-1}}}
\end{array}
$$

At $x=\infty$ we have

$$
\boldsymbol{\Gamma}_{n}(x)=G_{n}^{(\infty)}\left[\begin{array}{cc}
x^{n} & 0 \\
0 & x^{-\ell_{n}-1} \mathrm{e}^{V_{\text {sing, }}(x)}
\end{array}\right]\left(\mathbf{1}+\mathcal{O}\left(\frac{1}{x}\right)\right)
$$




$$
G_{n}^{(\infty)}=\left[\begin{array}{cc}
\frac{1}{\sqrt{h_{n}}} & 0 \\
0 & \sqrt{h_{n-1}}
\end{array}\right]
$$

Near any other pole $x=c$ of $V^{\prime}(x)$ we have

$$
\begin{array}{r}
\boldsymbol{\Gamma}_{n}(x)=G_{n}^{(c)}\left[\begin{array}{cc}
1 & 0 \\
0 & \mathrm{e}^{V_{\text {sing }, c}(x)}
\end{array}\right](\mathbf{1}+\mathcal{O}(x-c)) \\
G_{n}^{(c)}:=\left[\begin{array}{cc}
p_{n}(c) & \sqrt{h_{0}}(Q-c)_{n 0}^{-1} \mathrm{e}^{V_{\text {reg }, c}(c)} \\
r_{n-1}^{\star}(c) & \sqrt{h_{0}} c^{n-\ell_{n}+1}(Q-c)_{0 n-1}^{-1} \mathrm{e}^{V_{\text {reg }, c}(c)}
\end{array}\right]
\end{array}
$$

where in all these formulæ the notation $V_{\text {sing,p }}\left(V_{\text {reg, }, p}\right)$ denote the singular (regular) part of $V$ at the point $p$.

Near a hard-edge point $x=a$ we have 4

$$
\begin{array}{r}
\boldsymbol{\Gamma}_{n} \sim G_{n}^{(a)}\left[\begin{array}{cc}
1 & \pm \varkappa \ln (x-a) \\
0 & 1
\end{array}\right](\mathbf{1}+\mathcal{O}(x-a)) \\
G_{n}^{(a)}:=\left[\begin{array}{cc}
p_{n}(a) & \mathrm{e}^{V(a)} \mathcal{L}\left(\frac{p_{n}(z)-p_{n}(a)}{a-z}\right) \\
r_{n-1}^{\star}(a) & a^{n-1-\ell_{n}} \mathrm{e}^{V(a)} \mathcal{L}\left(\frac{r_{n}(z)-r_{n}(a)}{a-z}\right)
\end{array}\right]
\end{array}
$$

Together with these data we also have the jumps across the contours $\Gamma_{j}$ defining our moment functional: the situation in this respect is identical to 4 . In essence the matrix $\boldsymbol{\Gamma}_{n}(x)$ has the following jumps across the contour $\Gamma_{j}$

$$
\boldsymbol{\Gamma}_{n}(x)_{+}=\boldsymbol{\Gamma}_{n}(x)_{-}\left[\begin{array}{cc}
1 & 2 i \pi \varkappa_{i} \\
0 & 1
\end{array}\right] .
$$

Note that these jumps can be interpreted -depending on the point of view- as the Stokes' multipliers of the problem near the singularities.

\subsection{Isomonodromic Tau Function}

Using the results of [4] we find that the Jimbo-Miwa-Ueno isomonodromic tau function [13] is given by the same differential formulæ in Thm. 7.2 provided that we substitute the spectral curve of the connection with the spectral curve of the connection in the traceless gauge. In our situation the trace of $\frac{1}{x} \mathcal{D}_{n}(x)$ is $V^{\prime}(x)+\frac{n-1-\ell_{n}}{x}$ so that we perform a scalar gauge transformation

$$
\mathcal{A}_{n}^{(J M U)}=\frac{1}{x} \mathcal{D}_{n}(x)-\frac{1}{2}\left(V^{\prime}(x)+\frac{n-1-\ell_{n}}{x}\right) \mathbf{1}_{2 \times 2}
$$

This implies that the spectral parameter $y_{J M U}$ has the following relation to the previously employed $y$;

$$
y_{J M U}=y+\frac{1}{2}\left(V^{\prime}(x)+\frac{n-1-\ell_{n}}{x}\right)
$$

This in turn implies that -up to multiplicative factors independent of the isomonodromic times-

$$
\begin{aligned}
\Delta_{n}^{\ell_{n}}= & \mathcal{F}[V] \tau_{J M U} \\
& \ln \mathcal{F}[V]=-\frac{1}{2} \sum_{c=\text { finite pole of } \widehat{V}^{\prime}} \underset{x=c}{\operatorname{res}} \widehat{V}_{\text {sing }, c}^{\prime}(x) \widehat{V}_{\text {reg }, c}(x) \\
& \widehat{V}^{\prime}(x):=V^{\prime}(x)+\frac{n-1-\ell_{n}}{x},
\end{aligned}
$$


where $\widehat{V}_{\text {sing,c }}^{\prime}\left(\widehat{V}_{\text {reg,c }}\right)$ denotes the singular (regular) part of $\hat{V}^{\prime}$ at the pole $c$.

Example 10.1 For example let us consider the case relevant to the problem of the probability of the longest increasing sequence of random letter in a word of fixed length [16]

$$
V(x)=-t x-\sum_{\alpha=1}^{M} k_{\alpha} \ln \left(\frac{x-r_{\alpha}}{x}\right)
$$

In this case a direct computation $\left(\ell_{n} \equiv 0\right)$ gives for $\mathcal{F}$ the following expression

$$
\ln \mathcal{F}=-\frac{t}{2} \sum_{\alpha=1}^{M} k_{\alpha} r_{\alpha}+\frac{n-1}{2} \sum_{\alpha=1}^{M} k_{\alpha} \ln \left(-r_{\alpha}{ }^{2}\right)+\frac{1}{2} \sum_{\alpha=1}^{M}{k_{\alpha}}^{2} \ln \left(-r_{\alpha}{ }^{2}\right)-\frac{1}{2} \sum_{\alpha=1}^{M} \sum_{\beta \neq \alpha} \ln \left(\frac{r_{\beta}-r_{\alpha}}{r_{\alpha} r_{\beta}}\right)^{k_{\alpha} k_{\beta}}
$$

which is the result obtained also in formula (3.76) [16] : note that in that formula $r_{0}=0$ and $k_{0}=$ $n-\sum_{\alpha=1}^{M} r_{\alpha}$ and a little of algebraic manipulation shows the equivalence. Moreover the signs inside the logarithms in (10-20) are in fact irrelevant since omitting them would amount to multiplying $\mathcal{F}$ by a constant independent of the isomonodromic times, and hence could be reabsorbed in the definition of $\tau_{J M U}$.

\section{Schlesinger Transformations}

From the asymptotics that the shift $n \mapsto n+1$ implemented by the matrices $R_{n}$ are -in the language of isomonodromic deformations- what is known as elementary Schlesinger transformations. Specifically the shift $n \mapsto n+1$ corresponds to the following two types of elementary Schlesinger transformations according of the type of move (circle or line) (refer to formulæ 10-6 and 10-8):

Circle move. The Schlesinger transformation adds one to the first entry of the formal monodromy at $\infty$ and subtracts one from the second entry of the formal monodromy at zero

Line move. The Schlesinger transformation adds one and subtracts one to the first and second entry (respectively) of the formal monodromy at infinity, leaving the formal monodromy at zero unchanged.

However we can obtain a third type of elementary Schlesinger transformation by considering two distinct sequences of LOPs corresponding to two (weakly increasing) sequences of $\left\{\ell_{n}\right\}$ 's. Suppose indeed that we consider another sequence of LOPs and the ensuing connection $x \partial_{x}-\widetilde{\mathcal{D}_{n}}(x)$ for some fixed $n$ where the only difference between the two pairs of LOPs is that one (or more) circle-moves have been replaced by a line-move (or vice-versa) along the chain for $n^{\prime} \leq n$ : the only difference in the formulas will be that $\tilde{\ell}_{n}=\ell_{n} \pm 1$. This is implemented by the "circle-to-line" transformation $\mathcal{T}_{n}$ 2-15 (suitably normalized).

$$
\left[\begin{array}{c}
p_{n} \\
r_{n-1}^{\star}
\end{array}\right]=\left[\begin{array}{cc}
a+\frac{b}{x} & \frac{c}{x} \\
\frac{d}{x} & \frac{e}{x}
\end{array}\right]\left[\begin{array}{c}
\hat{p}_{n} \\
\hat{r}_{n-1}^{\star}
\end{array}\right]
$$

where the coefficients $a, b, c, d, e$ above can be obtained explicitly in terms of shifted Töplitz determinants using the form of $\mathcal{T}_{n}$ (2-15) and the normalizations (4-5), and the polynomials $\hat{p}_{n}, \hat{r}_{n-1}^{\star}$ refer to the elements of the sequence of biorthogonal polynomials associated to the sequence $\left\{\hat{\ell}_{k}\right\}$ : such sequence differs from $\left\{\ell_{k}\right\}$ because $\hat{\ell}_{n}=\ell_{n}-1$, namely there is a $k_{0} \leq n$ such that $\hat{\ell}_{k}=\ell_{k}-1, \forall k: \quad k_{0} \leq k \leq n$.

We therefore add the following third type of transformations; 
Circle-to-line move. The Schlesinger transformation subtracts one to the second entry of the formal monodromy at $\infty$ and adds one to the second entry of the formal monodromy at zero.

This last type of transformation shows that the orthogonal polynomials on the line and the orthogonal polynomials on the circle are related by a sequence $(n-1)$ Schlesinger transformations and at each step the Laurent biorthogonal polynomials that are obtained are those appearing in the solution of integrable lattice hierarchies associated to elementary orbits $[9]$.

\section{Conclusion}

As a general "philosophy", it is acknowledged in the literature that KP tau functions and isomonodromic tau functions are often, if not always, related one to the other, in the sense that a KP (or Toda) tau function is an isomonodromic tau function for a suitably chosen isomonodromic deformation. In the case of orthogonal polynomials this relation was explored in [15] for some class and extended in [6, 4. In this paper, this relation has been confirmed once more for the particular generalized Toda systems associated to "nonstandard" minimal orbits of the Borel subgroup: the natural bridge between the Hamiltonian and isomonodromic treatment is provided by the solution of the inverse spectral problem in terms of biorthogonal Laurent polynomials. It is to be expected that, whenever a description or formulation of an integrable dynamical problem in terms of (bi/multiple-orthogonal) polyomials is available, then a suitable definition of the tau function for the associated isomonodromic problem should tie the Hamiltonian tau function with the isomonodromic one. For instance, in the case of the biorthogonal polynomials arising in the study of two-matrix models [5, 7] a natural isomonodromic deformation of a polynomial connection can be derived; however the connection is a highly resonant one and at present a definition of isomonodromic tau function for resonant deformations of connections is not available. However it is possible to formulate such a notion $[8$ and the connection can thus be positively be established.

As it is recalled in the appendix to follow, the Laurent orthogonal polynomials which we have investigated in the present paper are related to the solution of the inverse spectral problem for Toda-like systems associated to certain minimal (or elementary) irreducible orbits. There exist in fact other minimal orbits for which a treatment in terms of orthogonal polynomials of some sort is not readily and generally available, although inspection of specific examples leads to expect that it is possible to overcome the difficulty. It is our intention to pursue the topic in future publications.

\section{Appendix: minimal irreducible co-adjoint orbits}

As it was mentioned earlier, every $n \times n$ principal submatrix of the Hessenberg matrix $Q$ that defines recurrence relations (5-1) belongs to a $2 n-2$-dimensional co-adjoint orbit of the Borel subgroup $\mathbf{B}_{n}$ of invertible upper triangular matrices in $s l(n)$. However, not every low-dimensional co-adjoint orbit can be obtained this way. In this appendix, we give a description of all irreducible co-adjoint orbits of $\mathbf{B}_{n}$ in $s l(n)$ that have a minimal dimension $2 n-2$.

First, we introduce some notations. Let $\mathfrak{b}_{-}$be a subalgebra of lower triangular matrices in $\operatorname{sl}(n)$. Denote by $J$ an $n \times n$ shift matrix (1s on the first superdiagonal and 0s everywhere else) and let Hess $s_{n}=$ $J+\mathfrak{b}_{-}$denote a set of lower Hessenberg matrices. An element $Q \in H e s s_{n}$ is called reducible if it has a block upper triangular form $Q=\left[\begin{array}{cc}Q_{11} & Q_{12} \\ 0 & Q_{22}\end{array}\right]$, where $Q_{11}$ is a $k \times k$ matrix $(0<k<n)$. $Q$ is called irreducible otherwise.

Orbits of the co-adjoint action of $\mathbf{B}_{n}$ on $\mathrm{Hess}_{n}$ are given by

$$
\mathcal{O}_{Q_{0}}=\left\{J+\left(\operatorname{Ad}_{b} Q_{0}\right)_{\leq 0}: b \in \mathbf{B}_{n}\right\} .
$$


It is easy to see that if $\mathcal{O}_{Q_{0}}$ contains a reducible (resp. irreducible) element then every element of $\mathcal{O}_{Q_{0}}$ is reducible (resp. irreducible). Therefore it makes sense to talk about irreducible orbits of the co-adjoint action. Our main goal in this appendix is to prove the following

Theorem 13.1 An irreducible co-adjoint orbit of $\mathbf{B}_{n}$ in Hess $_{n}$ has a minimal dimension $(2 n-2)$ if and only if it contains an element $Q_{0}$ of the form

$$
Q_{0}=J+H+\sum_{\alpha=1}^{k} E_{i_{\alpha}, i_{\alpha-1}-\varepsilon_{\alpha-1}}
$$

where

1. $\varepsilon_{i} \in\{0,1\}$ and $\varepsilon_{0}=0$

2. $1=i_{0}<i_{1}-\varepsilon_{1} \leq i_{1}<i_{2}-\varepsilon_{2} \leq i_{2}<\cdots<i_{k-1}-\varepsilon_{k-1} \leq i_{k-1}<i_{k}=n$

3. $H=\sum_{\alpha \in\{1, \ldots, n\} \backslash\left\{i_{0}, \ldots, i_{k-1}\right\}} h_{\alpha} E_{\alpha \alpha}$.

$$
Q_{0}=\left[\begin{array}{ccccccccccc}
0 & 1 & & & & & & & & & \\
\vdots & \star & 1 & & & & & & & & \\
\vdots & & 0 & 1 & & & & & & & \\
1 & \cdots & 0 & \star & 1 & & & & & & \\
& & \vdots & 0 & \star & 1 & & & & & \\
& & \vdots & \vdots & & \star & 1 & & & & \\
& & \vdots & \vdots & & & \star & 1 & & & \\
& & 1 & 0 & \cdots & \cdots & \cdots & 0 & 1 & & \\
& & & & & & & \vdots & \star & 1 & \\
& & & & & & & & & \\
& & & & & & & \vdots & & \star & 1 \\
& & & & & & & 1 & 0 & \cdots & \star
\end{array}\right]
$$

[An example with $n=11, k=3, i_{1}=4, i_{2}=8, i_{3}=11, \epsilon_{1}=\epsilon_{3}=0, \epsilon_{2}=1$.]

Remark 13.1 The case of $H=h E_{n n}$ and $\varepsilon_{\alpha}=0(\alpha=1, \ldots, k-1)$ was studied in [9, [10]. It is orbits of this type that can be studied via associated LOPs of the type appearing in this paper. Note that, in this case, parameters $\ell_{j}$ that were used in the main body of the paper are related to $i_{\alpha}$ via

$$
\ell_{j}=\max \left\{i_{\alpha}: i_{\alpha}<j\right\} .
$$

An investigation of propeties of moment functionals connected with a more general minimal orbits described in Theorem 13.1 will appear elsewhere.

Define a staircase pattern $(I, \varepsilon)$ as a collection of pairs of indices

$$
(I, \varepsilon)=\left\{\left(i_{1}, 1\right),\left(i_{2}, i_{1}-\varepsilon_{1}\right), \ldots,\left(i_{k}=n, i_{k-1}-\varepsilon_{k-1}\right)\right\},
$$

where

$$
1=i_{0}<i_{1}-\varepsilon_{1} \leq i_{1}<i_{2}-\varepsilon_{2} \leq i_{2}<\cdots<i_{k-1}-\varepsilon_{k-1} \leq i_{k-1}<i_{k}=n .
$$


In what follows we will often use a notation

$$
j_{\alpha}=i_{\alpha-1}-\varepsilon_{\alpha-1} .
$$

We say that $Q \in \mathrm{Hess}_{n}$ has a staircase pattern $(I, \varepsilon)$ if

$$
Q_{i_{\alpha}, j_{\alpha}} \neq 0 \text { and } Q_{i j}=0 \text { for } i>i_{\alpha}, j<j_{\alpha+1}(\alpha=1, \ldots, k) .
$$

The set of all matrices in $\mathrm{Hess}_{n}$ that have a staircase pattern $(I, \varepsilon)$ will be denoted by $\operatorname{Hess}(I, \varepsilon)$. For example, if $I=\{2,3, \ldots, n\}$ and $\varepsilon=\{0,0, \ldots, 0\}$, then $\operatorname{Hess}(I, \varepsilon)$ coincides with the set of $n \times n$ Jacobi matrices. An immediate property of the set $\operatorname{Hess}(I, \varepsilon)$ is that it is stable under the co-adjoint action of $\mathbf{B}_{n}$, since corner entries $Q_{i_{\alpha}, j_{\alpha}}$ and the entries "under the staircase" $Q_{i j}=0 i>i_{\alpha}, j<j_{\alpha+1}$ have only zeroes to the left and below and, thus the former are being acted upon only by the diagonal part of $\mathbf{B}_{n}$ and the latter cannot be made non-zero by the co-adjoint action.

Let us fix a staircase pattern $(I, \varepsilon)$. To begin the proof of Theorem 13.1 we first employ the strategy used in [11] to study generic staircase orbits.

Lemma 13.1 If $Q \in \operatorname{Hess}(I, \varepsilon)$ then there exist $\tilde{Q} \in \mathcal{O}_{Q}$ such that

$$
\begin{gathered}
\tilde{Q}_{i_{\alpha} j_{\alpha}}=1 \\
\tilde{Q}_{i j_{\alpha}}=0 \quad\left(j_{\alpha}<i<i_{\alpha}\right) \\
\tilde{Q}_{i_{\alpha} j}=0 \quad\left(j_{\alpha}<j<i_{\alpha} \text { and } j \neq j_{\beta}: \beta<\alpha, j_{\beta}<i_{\alpha}\right) \\
(\alpha=1, \ldots, k) .
\end{gathered}
$$

Proof. First, we use a diagonal conjugation to reduce $Q$ to an element with all corner entries equal to 1: $Q \rightarrow A d_{D}^{*} Q=D^{-1}(Q-J) D+J$, where $D=\operatorname{diag}\left(d_{1}, \ldots, d_{n}\right)=D_{k} \cdots D_{1}$ with diagonal matrices $D_{\alpha}$ defined by

$$
\left(D_{\alpha}\right)_{i i}=\left\{\begin{array}{cc}
1 & i \neq i_{\alpha} \\
d_{i_{\alpha}}=\left(D_{\alpha-1}^{-1} \cdots D_{1}^{-1} Q D_{1} \cdots D_{\alpha-1}\right)_{i_{\alpha} j_{\alpha}} & i=i_{\alpha}
\end{array}\right.
$$

Next, we use the co-adjoint action induced by a sequence of elementary upper-triangular matrices (each depending only on one parameter only) to set as many as possible of the entries in rows and columns occupied by corner entries equal to zero. More precisely, to eliminate an $\left(i, j_{\alpha}\right)$-entry $\left(j_{\alpha} \leq i<i_{\alpha}\right)$ using the corner entry $\left(i_{\alpha}, j_{\alpha}\right)$, one employs $A d_{\left(1+Q_{i j_{\alpha}} E_{i i_{\alpha}}\right)}^{*}$. Similarly, to eliminate an $\left(i_{\alpha}, j\right)$-entry $\left(j_{\alpha}<j<i_{\alpha}\right)$, one uses $A d_{\left(1-Q_{i_{\alpha} j} E_{j_{\alpha} j}\right.}^{*}$. Note that when we write $Q_{i j_{\alpha}}$ (resp. $Q_{i_{\alpha} j}$ ), we refer to entries of the "current" value of $Q$, i.e. to the element that belongs to the orbit through the initial $Q$ and that has been obtain through the sequence of transformations already applied.

The order in which we apply these elementary transformations is defined as follows: we first set to zero the entries in the 1st column (going down the column), then in the $i_{1}$ st row (moving right), then in the $j_{2}$ nd column (moving down), then in the $i_{2}$ nd row (moving right) etc. Through the entire process, we want, for every $l<m$, to use an elementary matrix of the from $\mathbf{1}+x E_{l m}$ at most once. This means, in particular, that any $\left(i_{\alpha}, j_{\beta}\right)$-entry, where $\beta<\alpha$ and $j_{\beta}<i_{\alpha}$ cannot be touched, since a matrix of the form $\mathbf{1}+x E_{j_{\beta} i_{\alpha}}$ has already been used to eliminate the $\left(j_{\beta}, j_{\alpha}\right)$-entry. This explains why entries $\left\{\left(i_{\alpha}, j_{\beta}\right): \beta<\alpha, j_{\beta}<i_{\alpha}\right\}$ are excluded from the list of entries in (13-5). On the other hand, all non-corner entries that are in the list can be set to 0 , regardless of their initial values. Q.E.D.

Corollary 13.1 For each $Q \in H e s s(I, \varepsilon)$ the matrix entries specified in [13-5) are independent functions on $\mathcal{O}_{Q}$.

Proof. It suffices to notice that applying to $\tilde{Q}$ constructed in Lemma 13.1 elementary transformations of the same type that was used in its construction, but in the reverse order and with arbitrary parameters, one can obtain an element in $\mathcal{O}_{Q}$ with arbitrary nonzero values of the corner entries and arbitrary values of non-corner values specified in (13-5). Q.E.D. 
Lemma 13.2 If, for some $1 \leq \alpha<k, \varepsilon_{\alpha}>1$, then, for any $Q \in \operatorname{Hess}(I, \varepsilon), \operatorname{dim} \mathcal{O}_{Q}>2 n-2$.

Proof. Denote by $M(I, \varepsilon)$ the set of pairs of indices that appear in the list given in (13-5). In view of the corollary above, we only need to show that, under conditions of the Lemma, the number of elements in $M(I, \varepsilon)$ is greater than $2 n-2$. We will also show that, if $0 \leq \varepsilon_{\alpha} \leq 1$ for $\alpha=1, \ldots, k-1$, then $\# M(I, \varepsilon)=2 n-2$.

We will use an induction on $k$ and $n$. Clearly, if $k=1$ then $\varepsilon_{0}=0$ and $\# M(I, \varepsilon)=2 n-2$. Moreover, $\# M(I, \varepsilon)=2 n-2$ for any $k$, provided $\varepsilon_{\alpha}=0$ for all $\alpha$. Let now $k=2$ and $\epsilon_{1}>0$. We are looking for a number of elements in the set $\left\{(1,1), \ldots,\left(i_{1}, 1\right),\left(i_{1}, 2\right), \ldots,\left(i_{1}, i_{1}-1\right) ;\left(i_{1}-\varepsilon_{1}, i_{1}-\varepsilon_{1}\right), \ldots,\left(i_{1}-1, i_{1}-\right.\right.$ $\left.\left.\varepsilon_{1}, i_{1}-\varepsilon_{1}\right),\left(i_{1}+1, i_{1}-\varepsilon_{1}, i_{1}-\varepsilon_{1}\right), \ldots,\left(n, i_{1}-\varepsilon_{1}\right), \ldots\left(n, i_{1}-1\right),\left(n, i_{1}+1\right), \ldots,(n, n-1)\right\}$, which is equal to $2\left(i_{1}-1\right)+2\left(n-\left(i_{1}-\varepsilon_{1}\right)\right)-2=2\left(n+\varepsilon_{1}-2\right)\left\{\begin{array}{ll}=2 n-2 & \text { if } \varepsilon_{1}=1 \\ >2 n-2 & \text { if } \quad \varepsilon_{1}>1\end{array}\right.$.

For $k>2$, let $s$ be such that $j_{s}<i_{1} \leq j_{s+1}$. We first consider the case when there is no $r$ such that $j_{r}=i_{1}$. Then the set $M(I, \varepsilon) \backslash\left\{(1,1), \ldots,\left(i_{1}, 1\right),\left(i_{1}, 2\right), \ldots,\left(i_{1}, i_{1}-1\right)\right\}$ has the same cardinality as a set $M\left(I^{\prime}, \varepsilon^{\prime}\right)$, where

$\left(I^{\prime}, \varepsilon^{\prime}\right)=\left\{\left(i_{2}-j_{2}, 1\right),\left(i_{3}-j_{2}, j_{3}-j_{2}+1\right), \ldots,\left(i_{s}-j_{2}, j_{s}-j_{2}+1\right),\left(i_{s+1}-j_{2}, j_{s+1}-j_{2}\right), \ldots,\left(i_{k}-j_{2}, j_{k}-j_{2}\right)\right\}=$ $\left\{\left(i_{2}-i_{1}+\varepsilon_{1}, 1\right),\left(i_{3}-i_{1}+\varepsilon_{1}, i_{2}-i_{1}+\varepsilon_{1}-\left(\varepsilon_{2}-1\right)\right), \ldots,\left(i_{s}-i_{1}+\varepsilon_{1}, i_{s-1}-i_{1}+\varepsilon_{1}-\left(\varepsilon_{s-1}-1\right)\right)\right.$, $\left.\left(i_{s+1}-i_{1}+\varepsilon_{1}, i_{s}-i_{1}+\varepsilon_{1}-\varepsilon_{s}\right), \ldots,\left(i_{k}-i_{1}+\varepsilon_{1}, i_{k-1}-i_{1}+\varepsilon_{1}-\varepsilon_{k-1}\right)\right\}$, that is

$$
\begin{gathered}
n^{\prime}=i_{k-1}^{\prime}=i_{k}-i_{1}+\varepsilon_{1}, \\
i_{\alpha}^{\prime}=i_{\alpha+1}-i_{1}+\varepsilon_{1} \quad \alpha=1, \ldots, k-1
\end{gathered}
$$

and

$$
\varepsilon_{\alpha}^{\prime}=\varepsilon_{\alpha+1}-1 \quad(1 \leq \alpha \leq s-2), \quad \varepsilon_{\alpha}^{\prime}=\varepsilon_{\alpha+1} \quad(s-1 \leq \alpha \leq k-2) .
$$

If $s \geq 2$ then $\varepsilon_{1} \geq 1$ and $\varepsilon_{s}=i_{s}-j_{s}>i_{s}-i_{1} \geq s-1 \geq 1$, so that $\varepsilon_{s-1}^{\prime}=\varepsilon_{s} \geq 2$ and, by the induction hypothesis, $\# M\left(I^{\prime}, \varepsilon^{\prime}\right)>2\left(n-i_{1}+\varepsilon_{1}-1\right) \geq 2\left(n-i_{1}\right)$ and $\# M(I, \varepsilon)>2\left(i_{1}-1\right)+2\left(n-i_{1}\right)=2(n-1)$.

If $s=1$ then $\varepsilon_{\alpha}^{\prime}=\varepsilon_{\alpha+1}$ for $1<\alpha \leq k-2$ and

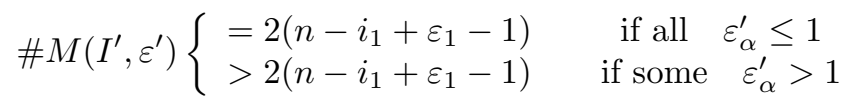

and thus, $\# M(I, \varepsilon)=2\left(i_{1}-1\right)+\# M\left(I^{\prime}, \varepsilon^{\prime}\right)$ is greater than $2 n-2$ if $\varepsilon_{\alpha}>1$ for some $\alpha>1$ and is equal to $2 n-2$ otherwise.

Finally, consider the case when $j_{r}=i_{1}$ for some $r>1$. If $r>2$ then $\varepsilon_{r}=i_{r}-j_{r}=i_{r}-i_{1} \geq r-1 \geq 2$. Define $(\tilde{I}, \tilde{\varepsilon})=(I, \varepsilon) \backslash\left\{\left(i_{2}, j_{2}\right), \ldots,\left(i_{r-1}, j_{r-1}\right)\right\}$. Then $(\tilde{I}, \tilde{\varepsilon})$ still defines an irreducible staircase pattern, $\tilde{k}=\#(\tilde{I}, \tilde{\varepsilon}), k$ and $\# M(I, \varepsilon)>\# M(\tilde{I}, \tilde{\varepsilon})>2 n-2$ by the induction hypothesis.

If $r=2$ then $\varepsilon_{1}=0, j_{2}=i_{1}$ and $\# M(I, \varepsilon)=2\left(i_{1}-1\right)+\# M\left(I^{\prime}, \varepsilon^{\prime}\right)$, where $\left(I^{\prime}, \varepsilon^{\prime}\right)=\left\{\left(i_{2}-i_{1}+\right.\right.$ $1,1),\left(i_{3}-i_{1}+1, i_{2}-i_{1}+1-\epsilon_{2}\right), \ldots,\left(n-i_{1}+1,\left(i_{k-1}-i_{1}+1-\epsilon_{k-1}\right)\right\}$ and, again by induction, the statement follows. Q.E.D.

We are now ready to complete the proof of Thm. 13.1

Proof of Theorem 13.1. Assume that $\operatorname{dim} \mathcal{O}_{Q}=2 n-2$. We have shown that if $Q \in \operatorname{Hess}(I, \epsilon)$ then $\epsilon_{\alpha} \leq 1$ for $\alpha=1, \ldots, k-1$. Assume that the latter condition is satisfied and consider the element $\tilde{Q}$ constructed in Lemma 13.1 Suppose that some non-corner entry $\tilde{Q}_{i j}(i>j)$ is nonzero. Then, by construction of $\tilde{Q}, j \neq j_{\alpha}(\alpha=1, \ldots, k)$. Define a diagonal matrix $D$ by

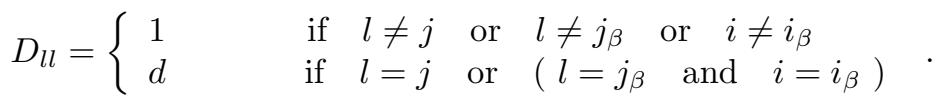

Then $\operatorname{Ad}_{D}^{*} \tilde{Q}=D^{-1} \tilde{Q} D$ has the same values as $\tilde{Q}$ in the entries specified by $13-5$ but $\left(\operatorname{Ad}_{D}^{*} \tilde{Q}\right)_{i j}=$ $d^{-1} \tilde{Q}_{i j}$. This means that the matrix entry $Q_{i j}$ viewed as a function on $\mathcal{O}_{Q}$ is independent of the matrix 
entries specified by (13-5), which is in contradiction with $\operatorname{dim} \mathcal{O}_{Q}=2 n-2$. Therefore, $\tilde{Q}_{i j}=0$ for all $(i, j) \neq\left(i_{\alpha}, j \alpha\right)$. Since, by Lemma $13.1 \tilde{Q}_{j_{\alpha} j_{\alpha}}=0$ for $\alpha=1, \ldots, k$, we proved that $\operatorname{dim} \mathcal{O}_{Q}=2 n-2$ implies that $\mathcal{O}_{Q}$ contains an element of the form (13-2).

To prove the converse consider an element $Q_{0}$ defined by (13-2). Clearly, for any $b \in \mathbf{B}_{n}, \operatorname{Ad}_{b}^{*} Q_{0}=$ $\operatorname{Ad}_{b}^{*}\left(Q_{0}-H\right)+H$, therefore it is sufficient to consider the case where $h=0$. In other words, we are interested in parametrizing the set

$$
\left\{\left(b\left(Q_{0}-H-J\right) b^{-1}\right)_{\leq 0}: b \in \mathbf{B}_{n}\right\}
$$

Note that, for $i>j$ we have

$$
\left(b E_{i j} b^{-1}\right)_{\leq 0}=\left(\left(b e_{i}\right)\left(e_{j}^{T} b^{-1}\right)\right)_{\leq 0}=\left(u v^{T}\right)_{\leq 0},
$$

where

$$
u=\left(\Pi_{i}-\Pi_{j-1}\right)\left(b e_{i}\right), v^{T}=\left(e_{j}^{T} b^{-1}\right)\left(\Pi_{i}-\Pi_{j-1}\right) .
$$

Thus,

$$
\left(b\left(Q_{0}-H-J\right) b^{-1}\right)_{\leq 0}=\sum_{\alpha=1}^{k}\left(u_{\alpha} v_{\alpha}^{T}\right)_{\leq 0}
$$

with

$$
u_{\alpha}=\left(\Pi_{i_{\alpha}}-\Pi_{i_{\alpha-1}-\varepsilon_{\alpha-1}-1}\right)\left(b e_{i_{\alpha}}\right), v_{\alpha}^{T}=\left(e_{i_{\alpha-1}-\varepsilon_{\alpha-1}}^{T} b^{-1}\right)\left(\Pi_{i_{\alpha}}-\Pi_{i_{\alpha-1}-\varepsilon_{\alpha-1}-1}\right) .
$$

Entries of vectors $u_{\alpha}, v_{\alpha}$ cannot be arbitrary. First,

$$
\left.v_{\alpha}^{T} u_{\alpha}=e_{j_{\alpha}}^{T} b^{-1}\left(\Pi_{i_{\alpha}}-\Pi_{j_{\alpha}-1}\right)\right) b e_{i_{\alpha}}=e_{j_{\alpha}}^{T} e_{i_{\alpha}}=0 .
$$

Next, if $\varepsilon_{\alpha}=0$, i.e. $j_{\alpha}=i_{\alpha-1}$, then

$$
\left(v_{\alpha}\right)_{j_{\alpha}}=\left(b^{-1}\right)_{j_{\alpha} j_{\alpha}}=\left(u_{\alpha-1}\right)_{j_{\alpha}}^{-1}
$$

Finally, if $\varepsilon_{\alpha}=1$, i.e. $j_{\alpha}=i_{\alpha-1}-1$, then

$$
v_{\alpha}^{T} u_{\alpha-1}=\left(v_{\alpha}\right)_{j_{\alpha}}\left(u_{\alpha-1}\right)_{i_{\alpha-1}-1}+\left(v_{\alpha}\right)_{j_{\alpha}+1}\left(u_{\alpha-1}\right)_{i_{\alpha-1}}=\left(b^{-1}\right)_{j_{\alpha} j_{\alpha}} b_{j_{\alpha} j_{\alpha}+1}+\left(b^{-1}\right)_{j_{\alpha} j_{\alpha}+1} b_{j_{\alpha}+1 j_{\alpha}+1}=0 .
$$

We claim that (13-7), (13-8), (13-9) are the only restrictions on $u_{\alpha}, v_{\alpha}$. We will verify this claim for $k=2$. The general case follows by an easy induction.

If $\epsilon_{1}=0$, we set $u_{1}=\operatorname{col}\left[u_{11}, u_{12}, u_{13}, 0, \ldots, 0\right]$ and $v_{1}^{T}=\left[v_{11}, v_{12}^{T}, v_{13}, 0, \ldots, 0\right]$, where $u_{11}, u_{13} \neq$ $0, v_{11} \neq 0, v_{13} \in \mathbb{C}$ and $u_{12}, v_{12} \in \mathbb{C}^{i_{1}-2}$. Similarly, $u_{2}=\operatorname{col}\left[0, \ldots, 0, u_{21}, u_{22}, u_{23}\right]$ and $v_{2}^{T}=\left[0, \ldots, 0, v_{21}=\right.$ $\left.u_{13}^{-1}, v_{22}^{T}, v_{23}\right]$, where $u_{21}, u_{23} \neq 0, v_{23} \in \mathbb{C}$ and $u_{22}, v_{22} \in \mathbb{C}^{n-i_{1}-1}$. We assume that conditions (13-7) are satisfied: $v_{1}^{T} u_{1}=v_{2}^{T} u_{2}=0$ and define

$$
b=\left(\begin{array}{ccccc}
v_{11}^{-1} & -v_{11}^{-1} v_{12}^{T} & u_{11} & 0 & 0 \\
0 & \mathbf{1} & u_{12} & 0 & 0 \\
0 & 0 & u_{13} & -u_{13} v_{22}^{T} & u_{21} \\
0 & 0 & 0 & \mathbf{1} & u_{22} \\
0 & 0 & 0 & 0 & u_{23}
\end{array}\right) \quad, \quad b^{-1}=\left(\begin{array}{ccccc}
v_{11} & v_{12}^{T} & v_{13} & * & * \\
0 & \mathbf{1} & -u_{12} u_{13}^{-1} & * & * \\
0 & 0 & v_{21} & v_{22}^{T} & v_{23} \\
0 & 0 & 0 & \mathbf{1} & -u_{22} u_{23}^{-1} \\
0 & 0 & 0 & 0 & u_{23}^{-1}
\end{array}\right) .
$$

The specified entries are consistent with the relation $b b^{-1}=\mathbf{1}$ and entries marked by $*$ s are uniquely determined by this relation.

Similarly, if $\epsilon_{1}=1$, we set $u_{1}=\operatorname{col}\left[u_{11}, u_{12}, u_{13}, u_{14}, 0, \ldots, 0\right]$ and $v_{1}^{T}=\left[v_{11}, v_{12}^{T}, v_{13}, v_{14}, 0, \ldots, 0\right]$, where $u_{11}, u_{13}, u_{14} \neq 0, v_{11} \neq 0, v_{13}, v_{14} \in \mathbb{C}$ and $u_{12}, v_{12} \in \mathbb{C}^{i_{1}-3} ;$ and $u_{2}=\operatorname{col}\left[0, \ldots, 0, u_{21}, u_{22}, u_{23}, u_{24}\right]$ 
and $v_{2}^{T}=\left[0, \ldots, 0, v_{21}, v_{22}=-v_{21} \frac{u_{13}}{u_{14}}, v_{23}^{T}, v_{24}\right]$, where $u_{21}, u_{22}, u_{24} \neq 0, v_{21} \neq 0, v_{24} \in \mathbb{C}$ and $u_{23}, v_{23} \in$ $\mathbb{C}^{n-i_{1}-2}$. Assuming again that $v_{1}^{T} u_{1}=v_{2}^{T} u_{2}=0$, define

$b=\left(\begin{array}{cccccc}v_{11}^{-1} & -v_{11}^{-1} v_{12}^{T} & -v_{11}^{-1} v_{13} & u_{11} & 0 & 0 \\ 0 & \mathbf{1} & 0 & u_{12} & 0 & 0 \\ 0 & 0 & v_{21}^{-1} & u_{13} & -v_{21}^{-1} v_{23}^{T} & u_{21} \\ 0 & 0 & 0 & u_{14} & 0 & u_{22} \\ 0 & 0 & 0 & 0 & \mathbf{1} & u_{23} \\ 0 & 0 & 0 & 0 & 0 & u_{24}\end{array}\right) \quad, \quad b^{-1}=\left(\begin{array}{cccccc}v_{11} & v_{12}^{T} & v_{13} & v_{14} & * & * \\ 0 & \mathbf{1} & 0 & -u_{12} u_{14}^{-1} & * & * \\ 0 & 0 & v_{21} & v_{22} & v_{23}^{T} & v_{24} \\ 0 & 0 & 0 & u_{14}^{-1} & 0 & -u_{22} u_{24}^{-1} \\ 0 & 0 & 0 & 0 & \mathbf{1} & -u_{23} u_{24}^{-1} \\ 0 & 0 & 0 & 0 & 0 & u_{24}^{-1}\end{array}\right)$

and observe that (13-7), (13-9) are consistent with $b b^{-1}=\mathbf{1}$ and entries marked by $*$ s can be uniquely determined .

To conclude the proof, observe that the right hand side of (13-6) is invariant under a transformation $u_{\alpha} \rightarrow t_{\alpha} u_{\alpha}, v_{\alpha} \rightarrow t_{\alpha}^{-1} v_{\alpha}$, where $t_{\alpha}$ are arbitrary non-zero parameters. Therefore, we can assume that

$$
\left(v_{\alpha}\right)_{j_{\alpha}}=1 \quad \text { if } \quad \alpha=1 \quad \text { or } \quad \varepsilon_{\alpha-1}=1
$$

Recall, that, if $\varepsilon_{\alpha-1}=0$, then $\left(v_{\alpha}\right)_{j_{\alpha}}$ is given by (13-8), while $\left(u_{\alpha}\right)_{j_{\alpha}}$ is determined by the condition (13-7) for all $\alpha$. Furthermore, if $\varepsilon_{\alpha-1}=1$, then $j_{\alpha}=i_{\alpha-1}+1$ and $\left(j_{\alpha}, j_{\alpha}\right),\left(j_{\alpha}+1, j_{\alpha}\right)$ and $\left(j_{\alpha}+1, j_{\alpha}+1\right)$-entries of the right hand side of (13-6) are given by

$$
\begin{aligned}
& \left(j_{\alpha}, j_{\alpha}\right): \quad\left(u_{\alpha-1}\right)_{j_{\alpha}}\left(v_{\alpha-1}\right)_{j_{\alpha}}+\left(u_{\alpha}\right)_{j_{\alpha}+1} \frac{\left(u_{\alpha-1}\right)_{j_{\alpha}}}{\left(u_{\alpha-1}\right)_{j_{\alpha}+1}}-\sum_{s=j_{\alpha}+2}^{i_{\alpha}}\left(u_{\alpha}\right)_{s}\left(v_{\alpha}\right)_{s} \\
& \left(j_{\alpha}+1, j_{\alpha}\right): \quad\left(u_{\alpha-1}\right)_{j_{\alpha}+1}\left(v_{\alpha-1}\right)_{j_{\alpha}}+\left(u_{\alpha}\right)_{j_{\alpha}+1} \\
& \left(j_{\alpha}+1, j_{\alpha}+1\right): \quad\left(u_{\alpha-1}\right)_{j_{\alpha}+1}\left(v_{\alpha-1}\right)_{j_{\alpha}+1}-\left(u_{\alpha}\right)_{j_{\alpha}+1} \frac{\left(u_{\alpha-1}\right)_{j_{\alpha}}}{\left(u_{\alpha-1}\right)_{j_{\alpha}+1}},
\end{aligned}
$$

where we have used (13-7), (13-9) and (13-9). Note that the entries in (13-10) are the only entries in (13-6) that depend on $\left(v_{\alpha-1}\right)_{j_{\alpha}},\left(v_{\alpha-1}\right)_{j_{\alpha}}$ and $\left(u_{\alpha}\right)_{j_{\alpha}+1}$. Moreover, (13-10) does not change under a transformation

$\left(u_{\alpha}\right)_{j_{\alpha}+1} \rightarrow\left(u_{\alpha}\right)_{j_{\alpha}+1}-t\left(u_{\alpha-1}\right)_{j_{\alpha}+1},\left(v_{\alpha-1}\right)_{j_{\alpha}} \rightarrow\left(v_{\alpha-1}\right)_{j_{\alpha}}+t,\left(v_{\alpha-1}\right)_{j_{\alpha}+1} \rightarrow\left(v_{\alpha-1}\right)_{j_{\alpha}}-t \frac{\left(u_{\alpha-1}\right)_{j_{\alpha}}}{\left(u_{\alpha-1}\right)_{j_{\alpha}+1}}$.

This means that we can set

$$
\left(u_{\alpha}\right)_{j_{\alpha}+1}=\left(u_{\alpha}\right)_{i_{\alpha-1}}=0 \quad \text { or } \quad \varepsilon_{\alpha-1}=1
$$

Under the normalizations (13-10), (13-12) and restrictions (13-7), (13-8), (13-9), the rest of the parameters in (13-6),

$$
\left(u_{\alpha}\right)_{s},\left(v_{\alpha}\right)_{s}, s=i_{\alpha-1}+1, \ldots, i_{\alpha}, \alpha=1, \ldots, k,
$$

can be chosen arbitrarily and, on the other hand, these parameters are uniquely determined by the right hand side of (13-6). Thus, for $Q_{0}$ satisfying conditions of Theorem 13.1 we have found an explicit parametrization of $\mathcal{O}_{Q_{0}}$ by $2 n-2$ independent parameters, which completes the proof. Q.E.D.

\section{References}

[1] M. Adler and P. van Moerbeke, "Generalized orthogonal polynomials, discrete KP and RiemannHilbert problems", Comm. Math. Phys. 207, 589-620, (1999).

[2] R. Beals, D. H. Sattinger, J. Szmigielski, "Multipeakons and the classical moment problem", Adv. Math. 154 229-257 (2000). 
[3] Y. M. Berezansky, "The integration of the semi-infinite Toda chain by means of inverse spectral problem", Rep. Math. Phys. 24 21-47 (1986).

[4] M. Bertola, B. Eynard, J. Harnad, "Semiclassical orthogonal polynomials, matrix models and isomonodromic tau functions", nlin.SI/0410043 submitted.

[5] M. Bertola, "Bilinear semi-classical moment functionals and their integral representation", J. App. Theory 121, 71-99 (2003).

[6] M. Bertola, B. Eynard and J. Harnad, "Partition functions for matrix models and isomonodromic tau functions", J. Phys. A: Math. Gen. 36, 3067-3083 (2003).

[7] M. Bertola, J. Harnad, B. Eynard, "Differential systems for biorthogonal polynomials appearing in 2-matrix models and the associated Riemann-Hilbert problem", Comm. Math. Phys. 243 no.2 (2003) 193-240.

[8] M. Bertola, M.Y. Mo, in preparation.

[9] L. Faybusovich, M. Gekhtman, "Elementary Toda orbits and integrable lattices", J. Math. Phys. 41 2905-2921

[10] L. Faybusovich, M. Gekhtman, "Inverse moment problem for elementary co-adjoint orbits.", Inverse Problems 17 (2001), no. 5, 1295-1306.

[11] M. Gekhtman, M. Shapiro, unpublished manuscript.

[12] L. Golinskii, P. Nevai, "Szegö difference equations, transfer matrices and orthogonal polynomials on the unit circle", Comm. Math. Phys. 223 (2001), no. 2, 223-259

[13] M. Jimbo, T. Miwa and K. Ueno, "Monodromy Preserving Deformation of Linear Ordinary Differential Equations with Rational Coefficients I.", Physica 2D, 306-352 (1981).

[14] M. Jimbo, T. Miwa, "Monodromy preserving deformation of linear ordinary differential equations with rational coefficients. II", Physica 2D (1981), 407-448.

[15] A.R. Its, A.V. Kitaev, and A.S. Fokas, "An isomonodromic Approach in the Theory of TwoDimensional Quantum Gravity", Usp. Matem. Nauk, 45, 6 (276), 135-136 (1990), (Russian), translation in Russian Math.Surveys, 45, no. 6, 155-157 (1990).

[16] A. R. Its, C. A. Tracy, H. Widom,"Random words, Toeplitz determinants and integrable systems. II", Advances in nonlinear mathematics and science. Phys. D 152/153 (2001), 199-224.

[17] F. Marcellán and I. A. Rocha, "Complex Path Integral Representation for Semiclassical Linear Functionals", J. Appr. Theory 94, 107-127 (1998).

[18] F. Marcellán and I. A. Rocha, "On semiclassical linear functionals: Integral representations", $J$. Comput. Appl. Math. 57 , 239-249 (1995) 